\title{
DNA Methylation Analysis of the Citrullus lanatus Response to Cucumber Green Mottle Mosaic Virus Infection by Whole-Genome Bisulfite Sequencing
}

\author{
Yuyan Sun, Min Fan * and Yanjun He \\ Institute of Vegetables, Zhejiang Academy of Agricultural Sciences, Hangzhou 310021, China; \\ syy1111@126.com (Y.S.); hyj1009@163.com (Y.H.) \\ * Correspondence: fanm@zaas.ac.cn; Tel.: +86-0571-8641-6057
}

Received: 25 March 2019; Accepted: 2 May 2019; Published: 7 May 2019

\begin{abstract}
DNA methylation is an important epigenetic mark associated with plant immunity, but little is known about its roles in viral infection of watermelon. We carried out whole-genome bisulfite sequencing of watermelon leaves at $0 \mathrm{~h}$ (ck), $48 \mathrm{~h}$, and 25 days post-inoculation with Cucumber green mottle mosaic virus (CGMMV). The number of differentially methylated regions (DMRs) increased during CGMMV infection and 2788 DMR-associated genes (DMGs) were screened out among three libraries. Most DMRs and DMGs were obtained under the $\mathrm{CHH}$ context. These DMGs were significantly enriched in the Kyoto Encyclopedia of Genes and Genomes (KEGG) pathways of secondary biosynthesis and metabolism, plant-pathogen interactions, Toll-like receptor signaling, and ABC transporters. Additionally, DMGs encoding PR1a, CaMs, calcium-binding protein, RIN4, BAK1, WRKYs, RBOHs, STKs, and RLPs/RLKs were involved in the watermelon-CGMMV interaction and signaling. The association between DNA methylation and gene expression was analyzed by RNA-seq and no clear relationship was detected. Moreover, downregulation of genes in the RdDM pathway suggested the reduced RdDM-directed $\mathrm{CHH}$ methylation plays an important role in antiviral defense in watermelon. Our findings provide genome-wide DNA methylation profiles of watermelon and will aid in revealing the molecular mechanism in response to CGMMV infection at the methylation level.
\end{abstract}

Keywords: watermelon; CGMMV; WGBS; DNA methylation; RNA-seq

\section{Introduction}

Cucumber green mottle mosaic virus (CGMMV) is a member of the Tobamovirus genus that produces typical mosaic patterning on infected plants. It was first reported in England in 1935 [1]. Since then, the diversity of CGMMV strains, including CV3, CV4, watermelon strain, Yodo strain, Indian C strain, and muskmelon strain have been found to cause serious diseases in cucurbits, diminishing their fruit yields and quality [1-5]. The disease cycle of CGMMV is complex, involving two viral dispersion components which occur in the field or conservation tillage systems and within seedling nurseries [6]. Over the past five years, CGMMV has spread rapidly and is now reported in more than 30 countries and regions including Australia and North America [7-11]. In CGMMV-infected watermelon, mottling and mosaicism develop on the leaves of young plants, brown necrotic lesions occur in the stems and peduncles, and sponginess, rotting, and dirty-red discolorations are seen in the flesh [6]. Currently, CGMMV management primarily relies on measures of cultural, biological, and host resistance [6]. Therefore, understanding the associated host response mechanism will aid in the development of potential control methods for CGMMV management.

Several studies have investigated interactions between cucurbits and CGMMV [12-18]. The transcriptome analysis of watermelon fruits and leaves identified genes that were differentially 
expressed in response to CGMMV infection, which were mainly involved in pathways of photosynthesis, plant-pathogen interactions, secondary metabolism, and plant hormone signal transduction [12,13]. Additionally, high-throughput microRNA (miRNA) sequencing analyses of CGMMV-infected cucumber and watermelon leaves revealed potential miRNAs that were activated in response to CGMMV infection [14,15]. Moreover, profiles of CGMMV-derived small interfering RNAs (siRNAs) in cucumber [16], bottle gourd [17], and watermelon [18] detected virus-derived siRNAs that were associated with interactions of CGMMV and cucurbits.

DNA methylation is an important epigenetic mark involved in genome stability, transcriptional inactivation, and environmental responses $[19,20]$. In plants, DNA methylation occurs in the sequence contexts of CG, CHG, and $\mathrm{CHH}(\mathrm{H}=\mathrm{A} / \mathrm{T} / \mathrm{C})$ [21]. Methylated CG (mCG) is catalyzed and maintained by methyltransferase 1 (MET1), while mCHG is catalyzed and maintained by chromomethylase 3 (CMT3) or CMT2 [22,23]. The maintenance of $\mathrm{mCHH}$ requires CMT2 and domains rearranged methyltransferases (DRM1 and DRM2) through the RNA-directed DNA methylation pathway, which require formation of double-stranded RNAs (dsRNAs) by two plant-specific homologs of RNA polymerase IV (Pol IV) and RNA-dependent RNA polymerase 2 (RDR2), then procession by Dicer-like 3 (DCL3) and Argonaute 4 (AGO4) or AGO6 to guide the DNA methylation [24]. The function of DNA methylation varies according to the genome location $[25,26]$. For example, DNA methylation of regulatory elements alters the chromatin structure, blocks transcriptional initiation, and downregulates gene expression. Conversely, methylation of repeat sequences is linked to silencing effects, and methylation of gene bodies is positively correlated with active transcription $[25,26]$.

The dynamics of DNA methylation are involved in plant growth and development as well as in plant response to environmental stresses. Studies have suggested that DNA methylation regulates leaf morphology, flowering time, fertility, embryogenesis, and seed development in plants [27-31]. Moreover, DNA methylation is associated with plant response to environmental stresses. For instance, whole-genome bisulfite sequencing of single-cell root hairs and multicellular stripped roots in response to heat stress in soybean showed hypomethylation after the heat stress [32]. In Arabidopsis, differentially methylated regions (DMRs) were observed in drought-exposed lineages compared with control plants, suggesting that DNA methylation is relatively stable under drought stress [33].

DNA methylation is also dynamically involved in the mechanism of plant immunity [34,35]. In Arabidopsis, enhanced defense responses to Pseudomonas syringae pv. tomato (Pst) DC3000 were observed when immune-response genes involved in DNA methylation and demethylation were mutated [36,37]. In wheat, DMRs with CHH hypomethylation were revealed following Blumeria graminis f. sp. tritici $(B g t)$ infection. Argonaute 4 was also significantly downregulated, resulting in a substantial reduction in AGO4a-sorted 24-nt siRNA levels, especially for genes located near transposable elements (TEs) [35]. Tomato yellow leaf curl virus (TYLCV) V2 interacts with its host HDA6 and interferes with the recruitment of MET1 by HDA6, resulting in decreased methylation of the viral DNA genome by transcriptional gene silencing with a concomitant increase in host susceptibility to TYLCV infection [38]. In tomato, Ty-1 confers resistance to geminiviruses by increasing cytosine methylation of the viral genomes, suggestive of enhanced transcriptional gene silencing [39].

RNA silencing (or RNA interference, RNAi) is a mechanism that induces messenger RNA (mRNA) degradation or inhibits translation at either the post-transcriptional level (PTGS) or the transcriptional level (TGS) which is involved in the RdDM pathway [40]. RNAi functions as an antiviral mechanism in plants and invertebrates [41]. Studies have shown that multiple DCLs, RDRs, and AGOs direct the antiviral RNAi defense in plants $[39,41,42]$. In addition, antiviral RNAi defense is suggested to mostly rely on a methylation-based defense, a process that involves the action of siRNA-directed methylation pathway component AGO4 [34,39,42].

Bisulfite sequencing has been used in analyzing DNA methylation in plant response to viral infection. For instance, reduced representation bisulfite sequencing (RRBS) of cucumber mosaic virus (CMV)-infected Nicotiana tabacum revealed that DMRs are enriched within CHH sequence contexts and are mainly located on the gene body [34]. Deep sequencing of bisulfite-treated DNA (BS-Seq) on 
TYLCV-infected tomato identify different epigenetic scenarios in the viral genome, suggesting that DNA methylation plays roles in plant defense and viral gene regulation [43]. Currently, whole-genome bisulfite sequencing (WGBS) has emerged as a powerful approach for studying DNA methylation by providing genome-wide methylation profiles at single-base resolution [44]. Whole-genome bisulfite sequencing has been successfully applied to profiling the DNA methylation in several plants, which reveals DNA methylation in controlling male sterility in Brassica napus [45], and regulating daily gene expression in Populus nigra and Populus trichocarpa [46,47]. However, WGBS application in studying DNA methylation dynamics in watermelon response to viral infection has not been reported. To investigate the profiles of DNA methylation and possible function of cytosine methylation in response to CGMMV infection in watermelon, we performed WGBS on watermelon leaves before and after CGMMV inoculation. Our findings identified DMRs and DMR-associated genes (DMGs) in response to CGMMV infection and reveal the possible role of DNA methylation in antiviral defense, which could be exploited to improve disease resistance in watermelon.

\section{Plants and Materials}

\subsection{Plant Materials and Cucumber Green Mottle Mosaic Virus Inoculation}

Seeds of the watermelon advanced inbred line "JJZ-M", which is susceptible to CGMMV, were planted in a greenhouse and kept at $25^{\circ} \mathrm{C}$. Seedlings at the two-true leaf stage were inoculated with CGMMV. Leaves were separately harvested at $0 \mathrm{~h}$ (ck), $48 \mathrm{~h}$ post-inoculation (hpi), and 25 days post-inoculation (dpi). Leaves from three plants were mixed as a sample. Presence of CGMMV was verified by RT-PCR using specific primers of the CGMMV coat protein (F: 5'-ATGGCTTACAATCC GATCACAC-3'; R: 5'-CTAAGCTTTCGAGGTGGTAGCC-3').

\subsection{DNA Extraction}

Genomic DNA samples were isolated from watermelon leaves using E.Z.N.A. ${ }^{\circledR}$ Tissue DNA Kit (Omega Bio-tek, Norcross, GA, USA) according to the manufacturer's instructions, and quality control was subsequently carried out on purified DNA samples. Genomic DNA was quantified using the TBS-380 fluorometer (Turner BioSystems Inc., Sunnyvale, CA, USA). High-quality DNA samples (OD $260 / 280=1.8-2.0,>6 \mu \mathrm{g}$ ) were used to construct the fragment library.

\subsection{Library Preparation and Illumina HiSeq Sequencing}

Before bisulfite treatment, $25 \mathrm{ng}$ lambda DNA was added to $5 \mu \mathrm{g}$ genomic DNA from watermelon leaves. The mixed DNA was then fragmented to $450 \mathrm{bp}$ with a sonicator (Sonics \& Materials Inc., Danbury, CT, USA). After blunt ending and the $3^{\prime}$-end addition of dA, Illumina methylated adapters were added according to the manufacturer's instructions by the Paired-End DNA Sample Prep Kit (Illumina, San Diego, CA, USA). The bisulfite conversion of genomic DNA was carried out using the ZYMO EZ DNA Methylation-Gold Kit (Zymo, Irvine, CA, USA), and amplified by 12 cycles of PCR using KAPA HiFi HotStart Uracil + ReadyMix (2×) (KAPA Biosystems, Boston, MA, USA). Ultra-high-throughput pair-end sequencing was carried out using Illumina HiSeq $X$ Ten according to the manufacturer instructions at Biozeron Biotechnology Co., Ltd. (Shanghai, China) and 150-bp paired-end reads were generated. Raw HiSeq sequencing data were processed by the Illumina base-calling pipeline (SolexaPipeline-1.0).

Whole-genome bisulfite sequencing data from this study can be accessed at sequence read archive (SRA) database from NCBI (https://www.ncbi.nlm.nih.gov/sra) with accession codes No.: SRR8797403 (ck), SRR8797402 (48 h), and SRR8797401 (25 d).

\subsection{Reads Quality Control and Mapping}

Raw paired-end reads were trimmed and quality controlled by Trimmomatic with default parameters. Clean BS-Seq reads were mapped to the reference genome of watermelon "Charleston 
Gray" with the Bisulfite Sequence Mapping Program (BSMAP) aligner allowing up to two mismatches to detect the methylation pattern of each cytosine in the genome [48]. The BSMAP uses the positions of all Cs in the reference sequences and applies bitwise masking to implement asymmetric $\mathrm{C} / \mathrm{T}$ transition: $T$ in bisulfite reads can be mapped to either $C$ or $T$ in the reference but not vice versa [48]. Alignments from both strands were combined, and for each read only the optimal alignments were kept. Multi-aligned reads, which were mapped to the watermelon genome at more than one location, were abandoned. Finally, the methylation status of each cytosine in watermelon genome was calculated on the basis of the alignments [49].

\subsection{Identification of Methylated Cytosine Sites}

The binomial test was performed for each cytosine base in the watermelon genome to check whether the cytosine site can be called a methylated cytosine site. Binomial probability values were then adjusted for multiple tests using false discovery rate (FDR). Cytosine sites with FDR $<0.01$ were defined as methylated cytosine sites, as described previously [49].

\subsection{Identification of Differentially Methylated Regions and DMR-Associated Genes}

The methylation level of each cytosine was defined as the proportion of reads showing $\mathrm{mC}$ among all reads covering the same cytosine. The methylation level of a region was defined as the average methylation level of all Cs in this region. Only cytosines covered with at least four reads in a library were considered to identify DMRs. DMRs were identified using a 200-bp sliding window with $50 \mathrm{bp}$ as a step-size. Cytosines (Cs) or thymines (Ts) were counted separately in each sliding window for three sequence contexts (CG, $\mathrm{CHG}$, or $\mathrm{CHH})$. The methylation level for a sliding window was determined as follows: methylation level $=\Sigma \mathrm{ai} /\left(\sum \mathrm{ai}+\mathrm{bi}\right)$, in which ai was the number of $\mathrm{Cs}$ and bi was the number of Ts mapping to the $C$ sites [35]. DNA methylation levels of different libraries were compared pairwise using Fisher's exact test, and p-values were adjusted for multiple comparisons using the Benjamini-Hochberg method. Windows with a FDR less than 0.05 and a change in methylation level more than 1.5-fold were retained for further analysis. The $\mathrm{p}$-value of each cytosine in selected regions was calculated by Fisher's exact test.

Differentially methylated cytosines (DMCs) were identified if $p \leq 0.01$ and FC $\geq 2$ with absolute methylation differences of $0.4,0.2$, or 0.1 for $C G, C H G$, or $\mathrm{CHH}$, respectively. Regions were only retained if they contained at least seven DMCs. Neighboring DMRs were combined if the gap was less than or equal to $100 \mathrm{bp}$ [50]. Genes overlapping with significant DMRs by at least $1 \mathrm{bp}$ in the functional region were defined as DMR-associated genes (DMGs) as described in a previous study [34]. In this study, seven functional regions, including intron, $2 \mathrm{~kb}$ upstream of genes (up-2k), $2 \mathrm{~kb}$ downstream of genes (down-2k), gene body, coding sequence (cds), $3^{\prime}$ untranslated region (utl3), and $5^{\prime}$ untranslated region (ut15), were divided based on gene structures of genome.

\subsection{Kyoto Encyclopedia of Genes and Genomes Pathway Analysis of DMR-Associated Genes}

Differentially methylated regions associated genes were tested for Kyoto Encyclopedia of Genes and Genomes (KEGG) pathway enrichment using the hypergeometric test (Fisher's exact test) with a 0.05 FDR correction. Pathways with $p<0.05$ were considered significantly enriched [51].

\subsection{Data Visualization}

Genome-wide expression and DNA methylation profiles were visualized in Integrative Genomics Viewer (IGV) [52].

\subsection{RNA Sequencing and Data Analysis}

To analyze the relationship between DNA methylation and gene expression, watermelon leaves were also collected for RNA extraction at $0 \mathrm{~h}, 48 \mathrm{~h}$, and $25 \mathrm{~d}$ post-CGMMV inoculation. Total RNA 
was extracted using TRIzol reagent (Invitrogen, Carlsbad, CA, USA) following the manufacturer's procedure. Total RNA was subjected to Poly(A) mRNA isolation using poly-T oligo-attached magnetic beads (Invitrogen). The mRNA was fragmented into small pieces and then reverse-transcribed to create the final cDNA library. The paired-end sequencing was carried out on an Illumina HiSeq 4000 platform. After the transcriptome data was generated. StringTie was used to determine expression levels for mRNAs by calculating fragments per kilobase of exon per million reads (FPKM) [53].

\section{Results}

\subsection{Phenotypic Observation of Watermelon Leaves Before and After Cucumber Green Mottle Mosaic} Virus Infection

At $25 \mathrm{dpi}$, characteristic symptoms of CGMMV, including mottling and mosaicism on the leaves and shriveling of veins, were observed in CGMMV-infected plants, while $48 \mathrm{~h}$-infected plants showed no obvious disease symptoms compared with control (ck) plants (Figure 1a). We further verified the presence of CGMMV using the RT-PCR analysis. Specific 486-bp fragments were amplified in both 48 hpi and 25 dpi plants (Figure 1b), which suggest CGMMV begun to accumulate at 48 hpi and highly accumulated at 25 dpi.

a

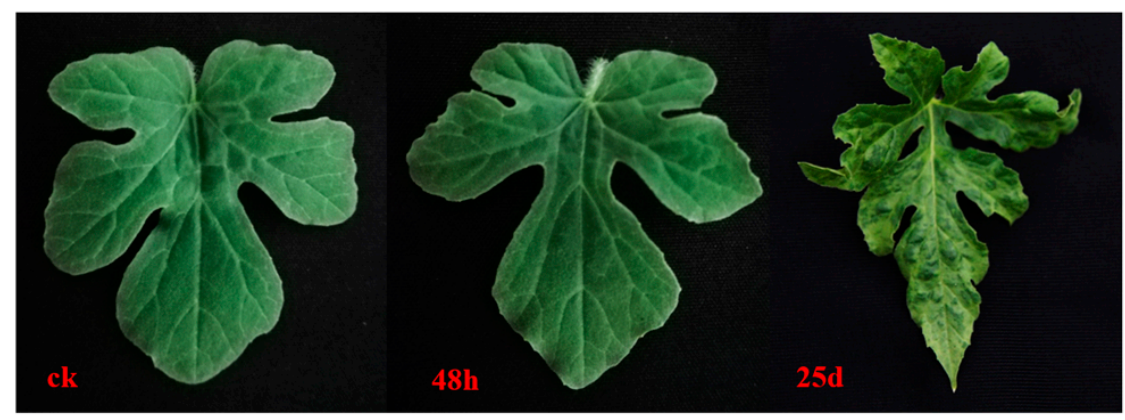

b

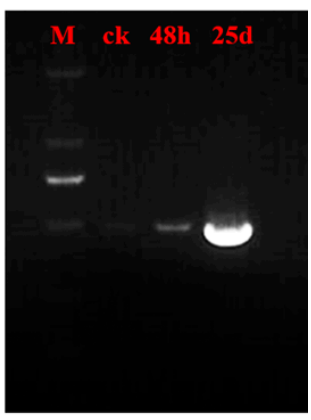

$1 \mathrm{~cm}$

Figure 1. Phenotypes and verification of watermelon leaves before and post Cucumber green mottle mosaic virus (CGMMV) infection. (a) Phenotypes of watermelon leaves before and after CGMMV infection. Leaves from left to right were ck (control), $48 \mathrm{~h}$, and $25 \mathrm{~d}$ post CGMMV infection, respectively. The scale bar is $1 \mathrm{~cm}$ for the watermelon leaves. (b) RT-PCR analysis of CGMMV presence for ck, $48 \mathrm{hpi}$ (hours post-inoculation), and $25 \mathrm{dpi}$ (days post-inoculation). Lanes from left to right were DL2000 marker, fragments of ck, $48 \mathrm{hpi}$, and $25 \mathrm{dpi}$, respectively.

\subsection{Profiles of Genome-Wide DNA Methylation in Watermelon Leaves}

Leaves of ck, $48 \mathrm{hpi}$, and 25 dpi plants were collected for the construction of bisulfite-treated genomic DNA libraries. In total, WGBS generated 91,070,116, 80,314,902, and 73,989,862 raw reads by paired-end sequencing for ck, $48 \mathrm{hpi}$, and $25 \mathrm{dpi}$ libraries, respectively, which obtained a total of $36,806,232,000$ raw bases and 32,900,506,657 clean bases for three libraries. Of the 83,839,958 clean reads from the ck library, $90.41 \%(75,798,928)$ were uniquely mapped to the reference genome of watermelon "Charleston Gray" (http://cucurbitgenomics.org/organism/4), while of the 71,659,074 clean reads from the 48 hpi library and 67,011,742 clean reads from the 25 dpi library, 89.35\% $(64,029,094)$ and $90.13 \%$ $(60,394,949)$ were uniquely mapped to the reference genome, respectively. Totals of $104,845,160,432$, and 150,563 clean reads with unknown bases (Ns) were obtained for the three libraries, respectively, and Q20 and Q30 percentages were approximately $97.0 \%$ and 90.0\%, respectively, for all three libraries. Coverage of sequencing data were $82.05 \%, 74.54 \%$, and $72.33 \%$ for ck, 48 hpi, and 25 dpi libraries. The CG percentages were 19.87\%, 19.79\%, and 20.29\%, average read depths were 12.06, 10.49, and 11.62 , and $60.73 \%, 51.57 \%$, and $54.15 \%$ cytosines were covered by at least four reads in the watermelon 
genome, respectively, for ck, $48 \mathrm{hpi}$, and $25 \mathrm{dpi}$ libraries. The depth and density of the sequencing were sufficient for high-quality genome-wide methylation analysis. Meanwhile, the bisulfite conversion efficiencies represented by the lambda DNA added into the libraries were $99.61 \%, 99.60 \%$, and $99.64 \%$ for ck, $48 \mathrm{hpi}$, and $25 \mathrm{dpi}$ libraries, respectively, indicating reliable results for the WGBS in the present study (Table 1).

Table 1. Summary of genome-wide methylation sequencing data

\begin{tabular}{cccc}
\hline Terms & ck & $\mathbf{4 8 ~ h p i ~}$ & 25 dpi \\
\hline Raw reads & $91,070,116$ & $80,314,902$ & $73,989,862$ \\
Raw bases & $13,660,517,400$ & $11,098,479,300$ & $12,047,235,300$ \\
Clean reads & $83,839,958$ & $71,659,074$ & $67,011,742$ \\
Clean bases & $12,402,189,963$ & $9,905,599,786$ & $10,592,716,908$ \\
Uniquely mapped reads & $75,798,928$ & $6,402,9094$ & $60,394,949$ \\
Uniquely mapped rates (\%) & 90.41 & 89.35 & 90.13 \\
Clean reads with unknown bases (Ns) & 104,845 & 160,432 & 150,563 \\
Quality score 20 (Q20) (\%) & 97.14 & 97.11 & 97.09 \\
Quality score 30 (Q30) (\%) & 90.84 & 90.82 & 90.80 \\
GC (\%) & 19.87 & 19.79 & 20.29 \\
Coverage (\%) & 82.05 & 74.54 & 72.33 \\
Strand depth & 12.06 & 10.49 & 11.62 \\
Depth >= 4 (\%) & 60.73 & 51.57 & 54.15 \\
Conversion rate (\%) & 99.61 & 99.60 & 99.64 \\
\hline
\end{tabular}

Genome-wide methylated cytosines totaled 276.95, 226.35, and 254.04 million for ck, 48 hpi, and 25 dpi libraries, respectively, by referring to the genome of watermelon "Charleston Gray" using BSMAP with a binomial test (Figure 2). Compared with ck, the number of methylated cytosines under CG, CHG, and $\mathrm{CHH}$ contexts decreased in the 48 hpi library, then increased in the 25 dpi library (Figure 2). Of note, CHG had the lowest methylated cytosines among all sequence contexts. In the ck library, the cytosine methylation percentage in the CHH context was similar to that in the CG context. However, the cytosine methylation percentage increased in the $\mathrm{CHH}$ context, while percentages of methylated cytosines in CG and CHG contexts decreased during CGMMV infection (Figure 3). In order to analyze the distribution of bases near methylated $C$ of non-CG sites and calculate the probability of methylated types, sequence characteristics of 9 bp bases neighboring methylated $\mathrm{CHG}$ and $\mathrm{CHH}$ were analyzed using WebLogo (http://weblogo.berkeley.edu/logo.cgi). Bases neighboring methylated C of CHG were $\mathrm{A}(\mathrm{T} / \mathrm{C}) \mathrm{G}$ and bases neighboring methylated $\mathrm{C}$ of $\mathrm{CHH}$ were $\mathrm{A}(\mathrm{T} / \mathrm{C}) \mathrm{A}(\mathrm{T} / \mathrm{C})$ in three libraries (Figure 4).

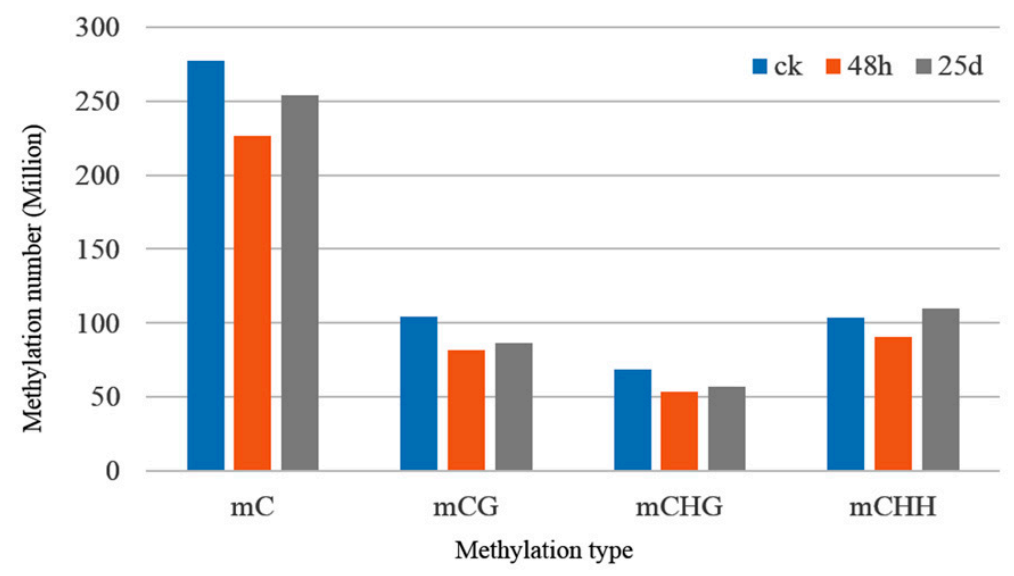

Figure 2. Numbers of $\mathrm{mC}, \mathrm{mCG}, \mathrm{mCHG}$ and $\mathrm{mCHH}$ identified in each sample by referring to the genome of watermelon "Charleston Gray". "m" stands for methylated in the bases: C, G, H (either A, T or $\mathrm{G})$ 


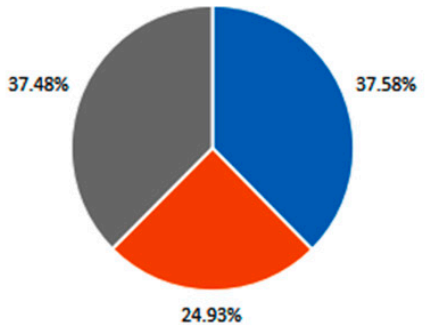

$\mathrm{ck}$

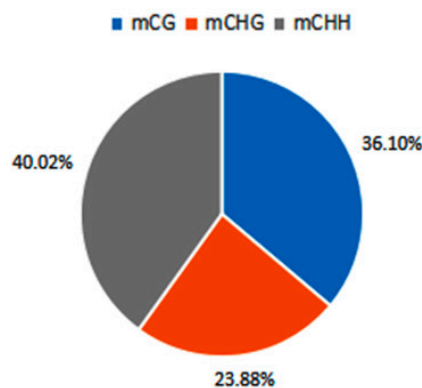

$48 \mathrm{~h}$

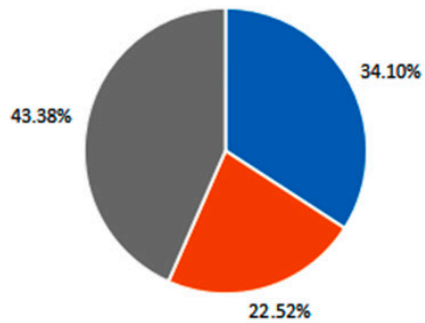

$25 \mathrm{~d}$

Figure 3. Percentages of methylated cytosines in each sample of watermelon leaves under each context.
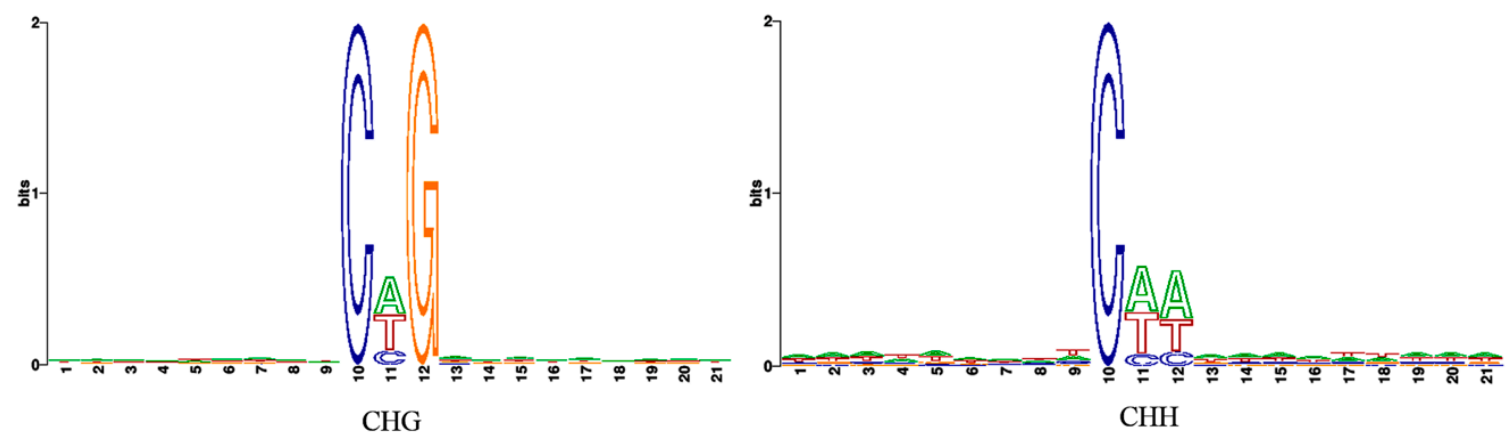

Figure 4. Sequence characteristic analysis of nine bp bases neighbouring methylated $\mathrm{CHG}$ and $\mathrm{CHH}$. The $x$-axis represents the nine bp upstream and downstream of $\mathrm{CHG}$ and $\mathrm{CHH}$, the height of the $y$-axis is the maximum entropy for the given sequence type (A, T, C, and $G$ ).

To present the global DNA methylation profiles of the three libraries, we further analyzed methylation levels and found them to be unevenly distributed throughout the watermelon chromosomes under CG, CHG, and CHH contexts (Figure 5). Dividing the genome into seven functional regions based on gene structures, including the gene body, $5^{\prime}$ untranslated region (utl5), coding sequence (cds), intron, $3^{\prime}$ untranslated region (utl3), $2 \mathrm{k}$ upstream (up-2k), and $2 \mathrm{k}$ downstream (down-2k) of genes, revealed the highest methylation level for up-2k and lowest for utl5 under $\mathrm{CHG}$ and $\mathrm{CHH}$ contexts (Figure 6). However, under the CG context, the highest methylation was observed in intron, followed by up-2k, down-2k, gene body, cds, utl3, and ut15 (Figure 6).

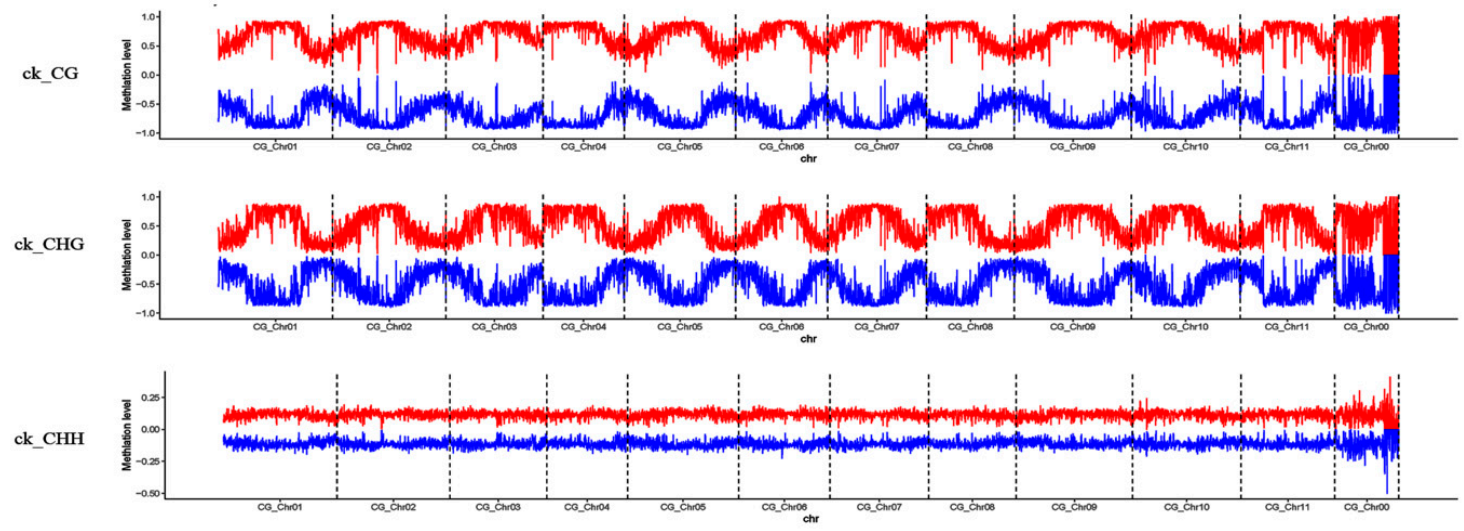

Figure 5. Cont. 

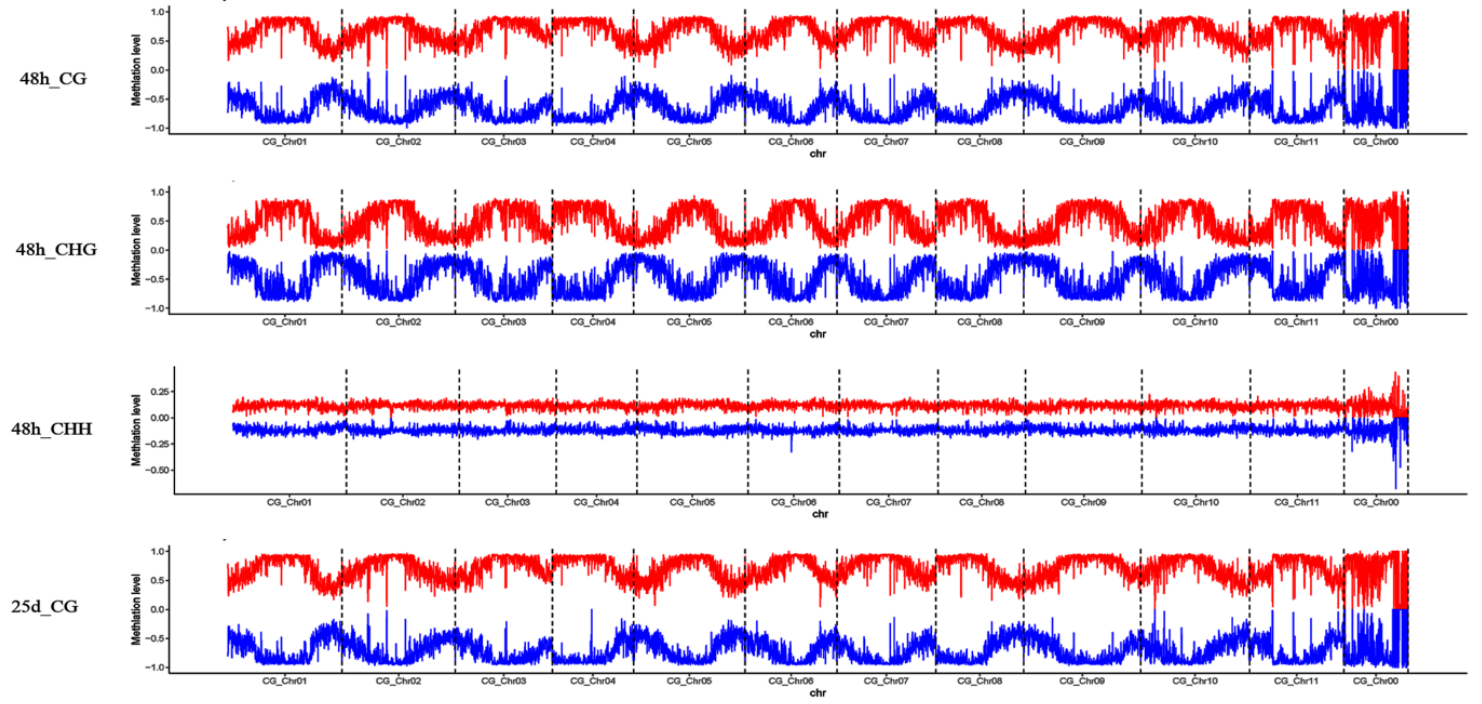

25d_CHG
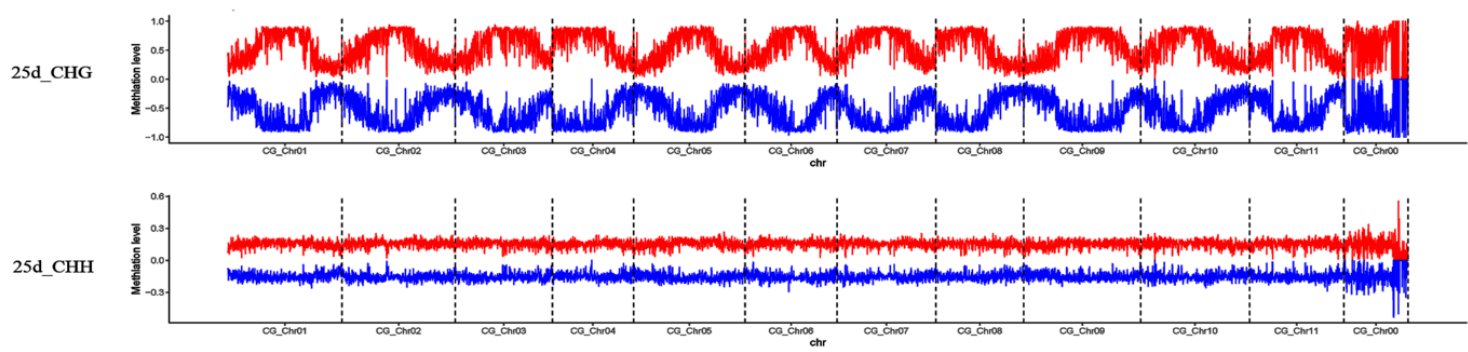

Figure 5. Chromosomal distribution of the global DNA methylation patterns in each sample under CG, CHG, and CHH contexts. CG_Chr00-CG_Chr11 in the $x$-axis represents chromosome $0-11$ of the watermelon "Charleston Gray". The $y$-axis represents the methylation level of DNA methylation patterns. The red lines indicate the methylation level in the sense strand of the watermelon genome and the blue lines indicate the methylation level in the antisense strand of the watermelon genome.
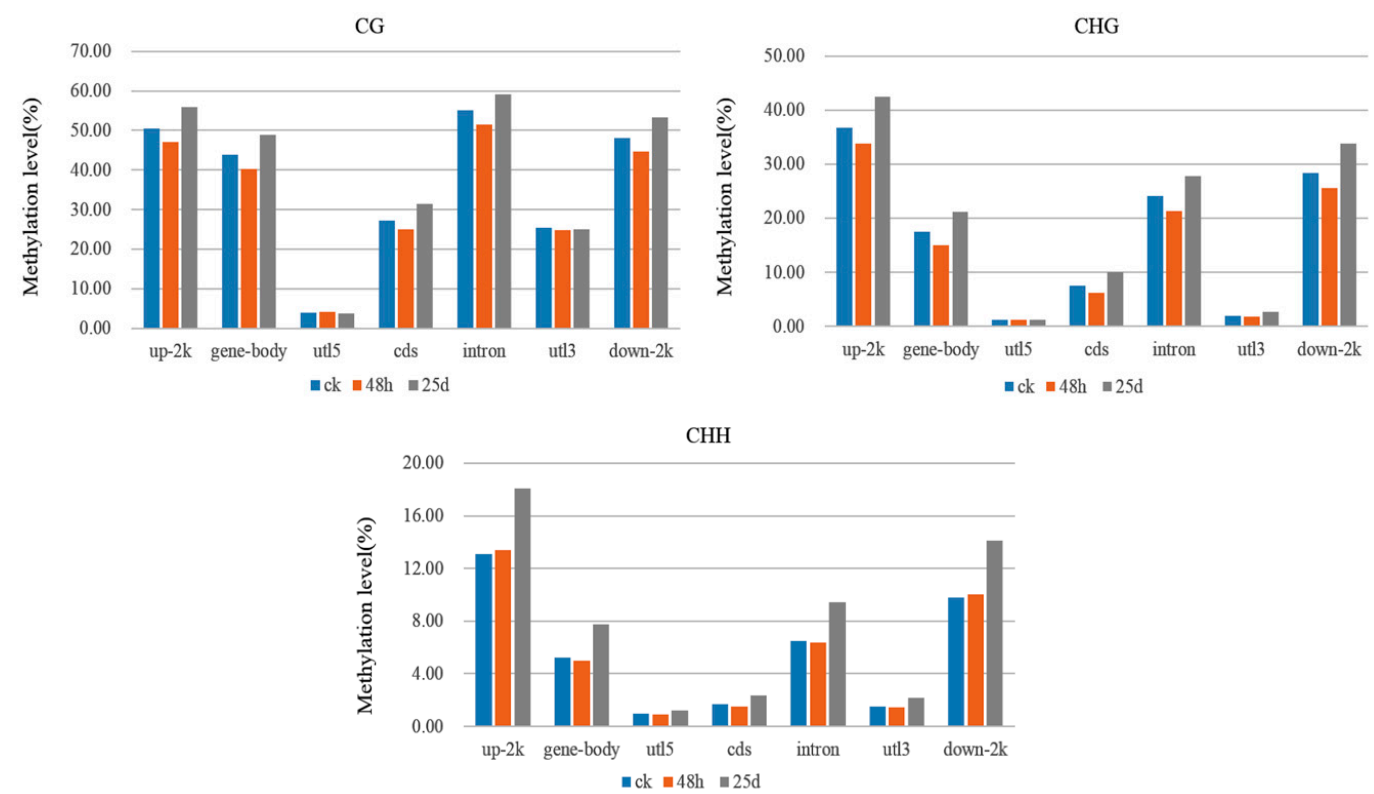

Figure 6. DNA methylation levels in different genomic functional regions of the watermelon genome. The $x$-axis represents the genomic regions in the watermelon genome. utl5: $5^{\prime}$ untranslated region, cds: coding sequence, utl3: $3^{\prime}$ untranslated region, up- $2 \mathrm{k}$ : $2 \mathrm{~kb}$ upstream of genes, down-2k: $2 \mathrm{~kb}$ downstream of genes. The $y$-axis represents the methylation levels for $\mathrm{ck}, 48 \mathrm{hpi}$, and $25 \mathrm{dpi}$ libraries under $\mathrm{CG}, \mathrm{CHG}$, and $\mathrm{CHH}$ contexts. 


\subsection{Detection of Differentially Methylated Regions}

Typically, the number of DMRs increased during CGMMV infection in watermelon leaves (Figure 7). Additionally, the number of hyper DMRs was highest in 25d_ck and lowest in 25d_48h, while hypo-DMRs were highest in 25d_48h and lowest in 25d_ck (Figure 7). We observed a total of 16,483 DMRs (10,683 hypermethylated and 5800 hypomethylated) for 48h_ck, 24,350 (23,086 hypermethylated and 1264 hypomethylated) for 25d_ck, and 30,523 (3949 hypermethylated and 26,574 hypomethylated) for 25d_48h (Table 2). Of these, 205, 205, and 16,073 were identified for 48h_ck, 61, 96, and 24,193 for 25d_ck, and 181, 215, and 30,127 for 25d_48h under CG, CHG, and CHH contexts, respectively (Table 2 and Table S1). Most DMRs were located in intergenic regions of the watermelon genome (Table 2 and Table S1). The fact that more DMRs were detected under the $\mathrm{CHH}$ context than under CG and CHG contexts implies that DNA methylation of $\mathrm{CHH}$ plays an important role in the CGMMV response.

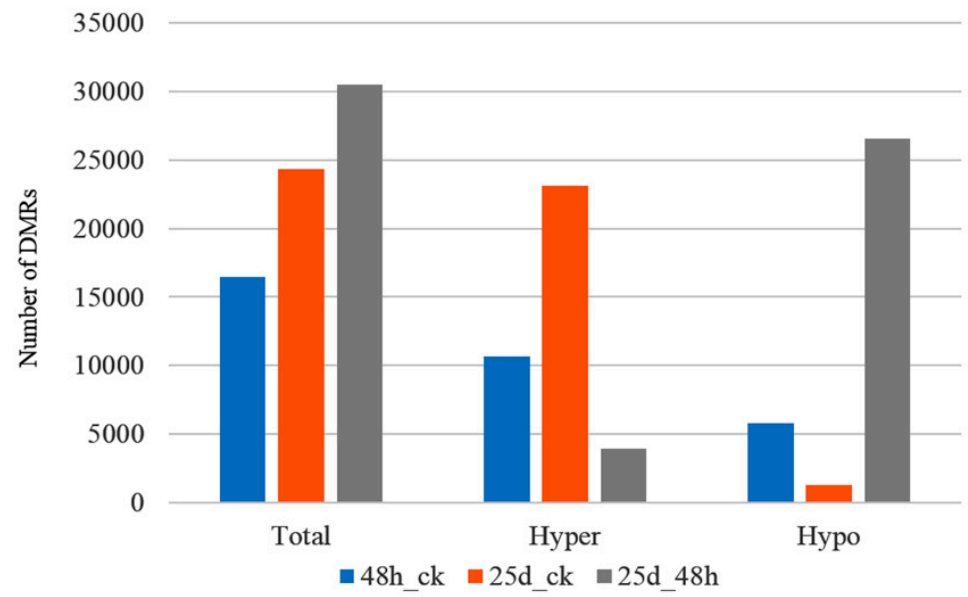

Figure 7. Number of differentially methylated regions (DMRs) identified in 48h_ck, 25d_ck, and 25d_48h.

Table 2. DMRs identified in watermelon.

\begin{tabular}{cccccccccc}
\hline Comparison & $\begin{array}{c}\text { DMRs } \\
\text { Total }\end{array}$ & $\begin{array}{c}\text { DMRs } \\
\text { Hyper }\end{array}$ & $\begin{array}{c}\text { DMRs } \\
\text { Hypo }\end{array}$ & $\begin{array}{c}\text { Intragenic } \\
\text { DMRs }\end{array}$ & $\begin{array}{c}\text { Intragenic } \\
\text { Hyper }\end{array}$ & $\begin{array}{c}\text { Intragenic } \\
\text { Hypo }\end{array}$ & $\begin{array}{c}\text { Intergenic } \\
\text { DMRs }\end{array}$ & $\begin{array}{c}\text { Intergenic } \\
\text { Hyper }\end{array}$ & $\begin{array}{c}\text { Intergenic } \\
\text { Hypo }\end{array}$ \\
\hline 48h_ck_CG & 205 & 23 & 182 & 39 & 11 & 28 & 166 & 12 & 154 \\
48h_ck_CHG & 205 & 29 & 176 & 41 & 11 & 30 & 164 & 18 & 146 \\
48h_ck_CHH & 16,073 & 10,631 & 5442 & 1293 & 860 & 433 & 14,790 & 9771 & 5009 \\
48h_ck_Total & 16,483 & 10,683 & 5800 & 1373 & 882 & 491 & 15,120 & 9801 & 5309 \\
25d_ck_CG & 61 & 19 & 42 & 11 & 3 & 8 & 50 & 16 & 34 \\
25d_ck.CHG & 96 & 63 & 33 & 31 & 21 & 10 & 65 & 42 & 23 \\
25d_ck_CHH & 24,193 & 23,004 & 1189 & 2112 & 2021 & 91 & 22,081 & 20,983 & 1098 \\
25d_ck_Total & 24,350 & 23,086 & 1264 & 2154 & 2045 & 109 & 22,196 & 21,041 & 1155 \\
25d_48h_CG & 181 & 28 & 153 & 28 & 6 & 22 & 153 & 22 & 131 \\
25d_48h_CHG & 215 & 12 & 203 & 36 & 3 & 33 & 179 & 9 & 170 \\
25d_48h_CHH & 30,127 & 3909 & 26,218 & 2502 & 292 & 2210 & 27,625 & 3617 & 24,008 \\
25d_48h_Total & 30,523 & 3949 & 26,574 & 2566 & 301 & 2265 & 27,957 & 3648 & 24,309 \\
\hline
\end{tabular}

\subsection{Detection of DMR-Associated Genes}

A total of 2788 DMGs were screened out among three libraries, including 863 for $48 \mathrm{~h} \_c k, 1520$ for 25d_ck, and 1691 for 25d_48h (Figure 8a). A total of 222 DMGs were common to all three libraries, 130 were common to $48 \mathrm{~h} \_c k$ and 25d_ck, 163 were common to $48 \mathrm{~h} \_c k$ and $25 \mathrm{~d} \_48 \mathrm{~h}$, and 549 were common to 25d_ck and 25d_48h (Figure 8a). Additionally, 32 (seven hyper and 25 hypo), 35 (10 hyper and 25 hypo), and 806 (561 hyper and 245 hypo) DMGs were identified for 48h_ck, eight (two hyper and six hypo), 24 (18 hyper and six hypo), and 1493 (1475 hyper and 18 hypo) DMGs were identified for 25d_ck, and 25 (four hyper and 21 hypo), 31 (31 hypo), and 1652 (58 hyper and 1594 hypo) DMGs were identified for 25d_48h under the contexts of CG, CHG, and CHH, respectively (Figure 8b-d; 
Table S2). Consistent with DMR findings, many more DMGs were detected under the CHH context than under CG and CHG contexts.

a

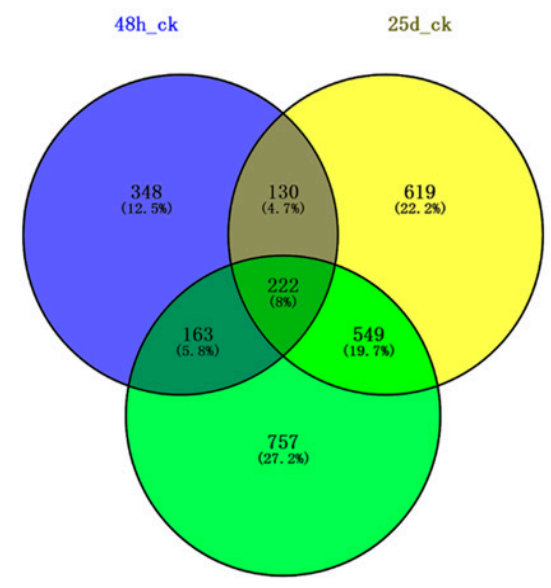

25d_48h

C

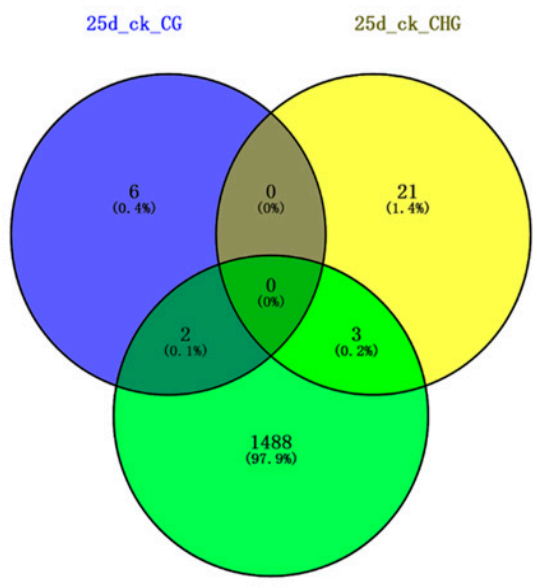

25d_ck_CHH b

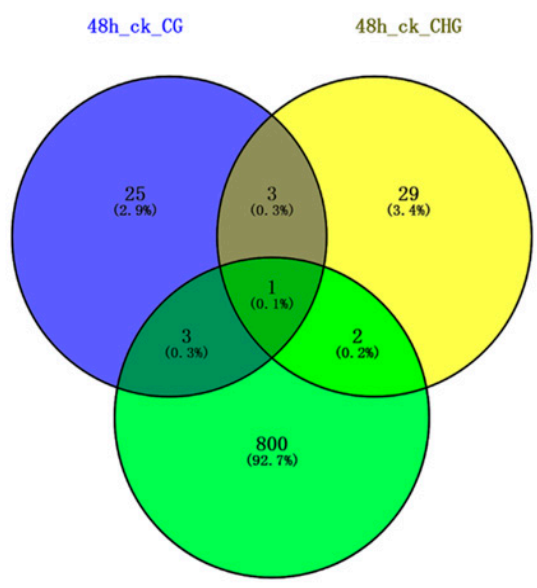

48h_ck_CHH

d

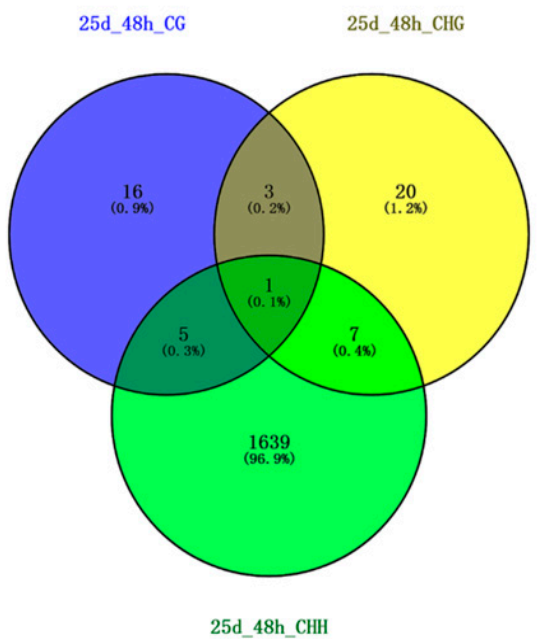

Figure 8. Venn diagram of DMR-associated genes (DMGs) among three libraries. (a) Venn diagram of DMGs among 48h_ck, 25d_ck, and 25d_48h; (b) Venn diagram of DMGs for 48h_ck under CG, CHG, and CHH contexts; (c) Venn diagram of DMGs for 25d_ck under CG, CHG, and CHH contexts; (d) Venn diagram of DMGs for 25d_48h under CG, CHG, and CHH contexts.

\subsection{Functional Analysis of DMR-Associated Genes}

A total of 196, 234, and 232 KEGG pathways for DMGs were identified in 48h_ck, 25d_ck, and 25d_48h libraries, respectively (Table S3). Among these, 14, 23, and 21 KEGG pathways with $p<0.05$ were significantly enriched, respectively (Figure 9 and Table S4). For example, phenylalanine metabolism (ko00360), phenylpropanoid biosynthesis (ko00940), cutin, suberine, and wax biosynthesis (ko00073), linoleic acid metabolism (ko00591), the Toll-like receptor signaling pathway (ko04620), starch and sucrose metabolism (ko00500), plant-pathogen interactions (ko04626), and ABC transporters (ko02010) were significantly enriched during CGMMV infection (Figure 9 and Table S3). 

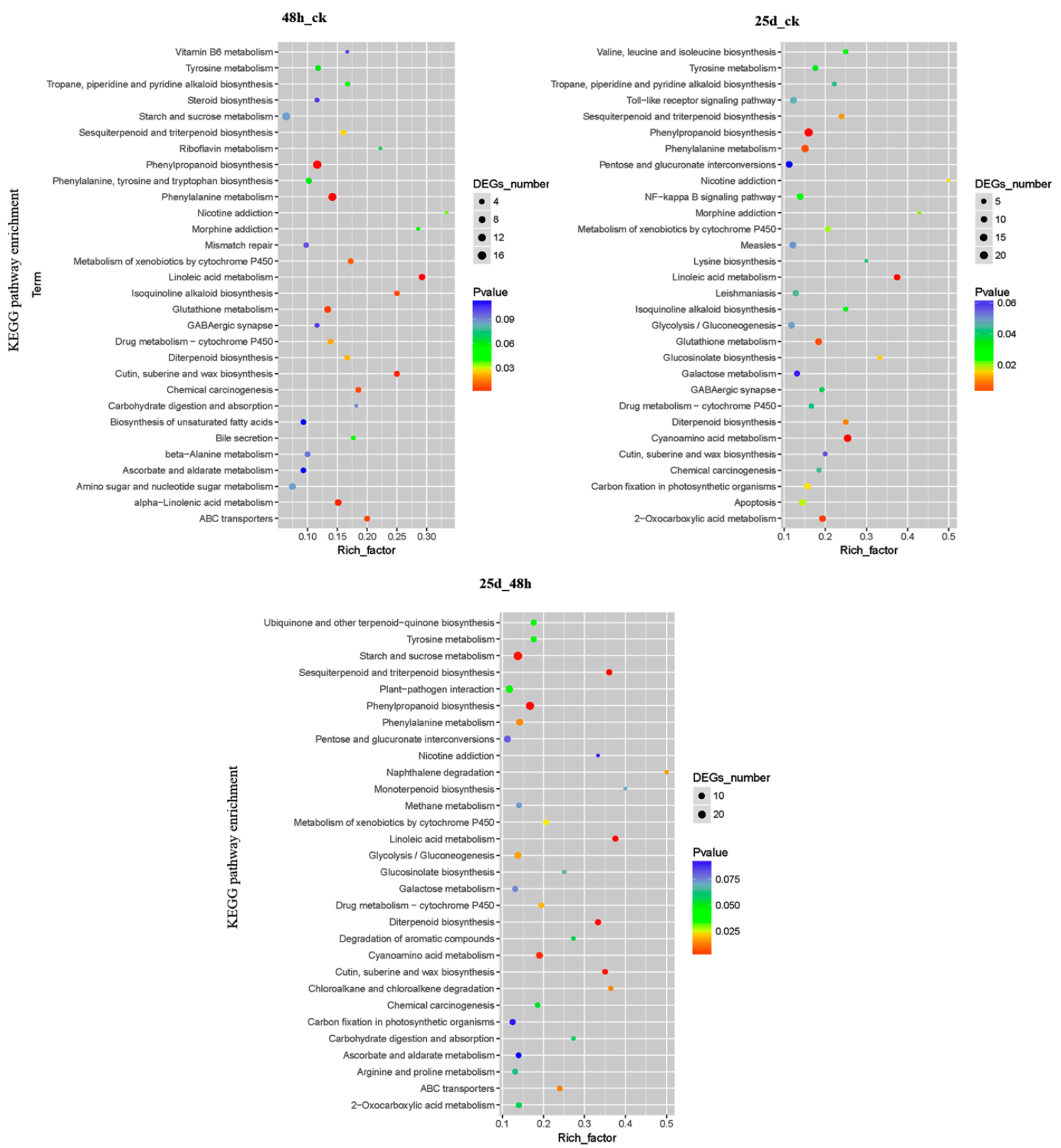

Figure 9. Statistics of the top 20 pathways enriched for DMGs in $48 \mathrm{~h}$ ck, 25d_ck, and 25d_48h. The size of each circle represents the number of DMGs enriched in the corresponding pathway. The enrichment factor was calculated using the number of enriched genes divided by the total number of background genes in the corresponding pathway. The $p$-value was calculated using the Benjamini-Hochberg correction. A pathway with $p<0.05$ was considered significantly enriched.

3.6. DMR-Associated Genes Related to the Cucumber Green Mottle Mosaic Virus Response in Watermelon Leaves

In this study, a few DMGs were invovled in the KEGG pathways of secondary biosynthesis and metabolism, plant-pathogen interactions, Toll-like receptor signaling, and $A B C$ transporters. Among them, 15 DMGs, encoding proteins of sucrose synthase, 4-coumarate-CoA ligase, linoleate 9S-lipoxygenase, pectinesterase, WRKY, calmodulin-like protein, pathogenesis-related protein, RPM1-interacting protein, brassinosteroid insensitive 1-associated receptor kinase, and ABC transporter were selected for visualization the dynamics of genic DNA methylation using the IGV (Figure S1). 
3.6.1. DMR-Associated Genes Involved in Secondary Biosynthesis and Metabolism

A subset of DMGs were involved in the pathways of secondary biosynthesis and metabolism, including phenylpropanoid biosynthesis and metabolism (ko00360; ko00940), cutin, suberine and wax biosynthesis (ko00073), linoleic acid metabolism (ko00591), and starch and sucrose metabolism (ko00500) (Table S4). Most of these DMGs were hypermethylated for 25d_ck and hypomethylated for 25d_48h (Table S4).

A total of 43 DMGs were involved in phenylpropanoid biosynthesis (ko00940) and phenylpropanoid metabolism (ko00360) pathways (Table S4). Of these, four DMGs (ClCG09G003320, ClCG10G012970, ClCG10G012990, and ClCG10G013000) encoding cinnamoyl-CoA reductase (CCR) were identified. Both ClCG09G003320 (CCR1) and ClCG10G012970 (CCR2) were hypermethylated for 25d_ck and hypomethylated for 25d_48h under the CHH context. ClCG10G012990 (CCR2) was hypomethylated for 48h_ck under the CHH context. Another DMG (ClCG10G013000) encoding CCR2 was hypermethylated for $48 \mathrm{~h} \_\mathrm{ck}$ and hypomethylated for $25 \mathrm{~d} \_48 \mathrm{~h}$ under the $\mathrm{CHH}$ context. Additionally, two DMGs (ClCG07G002280 and ClCG11G012560) encoded 4-coumarate-CoA ligase. ClCG07G002280 was hypermethylated for 25d_ck and hypomethylated for 25d_48h under the CHH context, and ClCG11G012560 was hypomethylated for $25 \mathrm{~d} \_48 \mathrm{~h}$ under the $\mathrm{CHH}$ context. Moreover, 17 DMGs encoding peroxidase were identified in this pathway, of which most were hypermethylated or hypomethylated under the $\mathrm{CHH}$ context.

Eight DMGs (ClCG01G012910, ClCG02G022090, ClCG03G000870, ClCG04G001450, ClCG05G002780, ClCG05G002790, ClCG06G000770, and ClCG11G009050) were associated with cutin, suberine, and wax biosynthesis pathways (Table S4). These DMGs encoded fatty acyl-CoA reductase (FAR), cytochrome P450 (CYP), protein ECERIFERUM 1-like (CER1-like) and omega-hydroxypalmitate O-feruloyl transferase. ClCG03G000870 (CYP), ClCG11G009050 (omega-hydroxypalmitate O-feruloyl transferase), and ClCG06G000770 (CER1-like) were hypomethylated for 25d_48h under the CHH context, ClCG04G001450 (FAR2) was hypomethylated for 48h_ck under the CHH context, and ClCG05G002780 (CER1-like) was hypermethylated for 25d_ck and hypomethylated for 25d_48h under the CHH context. Two FAR3 genes (ClCG01G012910 and ClCG02G022090) were commonly hypermethylated for 48h_ck and 25d_ck, and hypomethylated for 25d_48h under the $\mathrm{CHH}$ context.

Among the 13 DMGs involved in the linoleic acid metabolism pathway, 11 DMGs (ClCG02G015640, ClCG02G015670, ClCG02G015680, ClCG02G015690, ClCG02G015700, ClCG02G015710, ClCG02G015730, ClCG02G015750, ClCG02G023560, ClCG02G023610, and ClCG09G007920) encoded lipoxygenases (LOXs) (Table S4). In all cases under the CHH context, ClCG02G015640 (linoleate 9S-LOX6-like) was hypermethylated for 25d_ck, and ClCG02G023610 (LOX7) was hypermethylated for 25d_48h. ClCG02G023560 (linoleate 13S-LOX2-1) and ClCG09G007920 (linoleate 9S-LOX-like) were hypermethylated for 25d_ck, ClCG02G015690 (linoleate 9S-LOX5) was hypermethylated for 48h_ck and 25d_ck, and ClCG02G015750 (linoleate 9S-LOX5 isoform X2) was hypermethylated for 48h_ck and 25d_48h. ClCG02G015680 and ClCG02G015700 encoding linoleate 9S-LOX6-like were hypermethylated for 25d_ck and hypomethylated for 25d_48h, ClCG02G015670 (LOX) was hypomethylated for 48h_ck and 25d_48h, ClCG02G015710 (linoleate 9S-LOX6-like) was hypermethylated for 25d_ck and hypomethylated for 48h_ck and 25d_48h, and ClCG02G015730 (linoleate 9S-LOX6-like isoform X1) was hypermethylated for $48 \mathrm{~h} \_c k$ and $25 \mathrm{~d} \_c k$ and hypomethylated for $25 \mathrm{~d} \_48 \mathrm{~h}$.

Forty DMGs were involved in the starch and sucrose metabolism pathway (ko00500) (Table S4). These mainly encoded pectinesterase (PE), hexokinase (HK), sucrose synthase (SUS), $\beta$-amylase, cellulose synthase (CesA), and $\beta$-glucosidase. Six DMGs (ClCG03G014780, ClCG05G007400, ClCG06G007740, ClCG07G008840, ClCG09G005530, and ClCG09G020720) encoded PEs, of which ClCG09G020720, ClCG03G014780 and ClCG07G008840 were hypermethylated for 48h_ck, 25d_ck, and 25d_48h under the CHH context. In all cases under the CHH context, ClCG05G007400 and ClCG09G005530 were hypomethylated for 48h_ck and 25d_48h, ClCG06G007740 encoding PE29 was hypermethylated for 25d_ck and hypomethylated for 25d_48h, and ClCG01G008480 encoding SUS5 was hypermethylated for 48h_ck and hypomethylated for 25d_48h. DMGs encoding $\beta$-amylase (ClCG07G011170) and CesA 
(ClCG10G011170) were commonly hypermethylated for 25d_ck and hypomethylated for 25d_48h, and DMGs encoding HK1 (ClCG11G002110) and HK2 (ClCG05G018300) were hypomethylated for 25d_48h and hypermethylated for $48 \mathrm{~h} \_c k$.

\subsubsection{DMR-Associated Genes Involved in Plant-Pathogen Interactions}

Twenty-two DMGs were identified as participating in plant-pathogen interactions (ko04626) (Table S4). These mainly encoded pathogenesis-related protein (PR), respiratory burst oxidase homolog protein $(\mathrm{RBOH})$, cyclic nucleotide-gated ion channel protein (CNGC), RPM1-interacting protein 4 (RIN4), BRASSINOSTEROID INSENSITIVE 1-associated receptor kinase 1 (BAK1), calmodulin (CaM)-like protein, calcium-binding protein, and WRKY transcription factors (TFs). Two DMGs (ClCG07G007870 and ClCG02G019200) encoding WRKY22 and WRKY26 were hypomethylated for 48h_ck and 25d_ck under the CHH context, while DMG (ClCG02G002690) encoding WRKY1 was hypermethylated for 25d_ck and hypomethylated for 25d_48h under the CHH context. ClCG02G005880 and ClCG02G016580 encoding CaM-like 1 and CaM-like 8 were hypomethylated for 25d_48h under CG and CHH contexts, calcium-binding protein CML22 (ClCG05G003310) was hypomethylated for 25d_48h and calcium-binding protein CML31 (ClCG03G008520) was hypomethylated for 48h_ck and 25d_48h under the CHH context. Three RBOHs (ClCG01G002290, ClCG03G017090, and ClCG07G007770) and a PR1a gene (ClCG02G007230) were hypomethylated for 25d_48h under the CHH context, while a BAK1 gene (ClCG09G015110) was hypermethylated for 48h_ck and an RIN4 gene (ClCG05G003210) was hypermethylated for $25 \mathrm{~d} \_c k$ and hypomethylated for $25 \mathrm{~d} \_48 \mathrm{~h}$ under the $\mathrm{CHH}$ context.

\subsubsection{DMR-Associated Genes Involved in the Toll-like Receptor Signaling Pathway}

Fourteen DMGs were involved in the Toll-like receptor signaling pathway (ko04620) (Table S4). Four of these (ClCG02G020720, ClCG07G002710, ClCG09G010710, and ClCG11G011240) encoded serine/threonine-protein kinases (STKs). ClCG02G020720 was hypermethylated for 25d_ck under the $\mathrm{CHH}$ context and ClCG07G002710 was hypomethylated for 48h_ck under the CG context. ClCG09G010710 and ClCG11G011240 were hypermethylated for 25d_ck and hypomethylated for 25d_48h under the CHH context. Six DMGs (ClCG05G016550, ClCG05G017520, ClCG05G025640, ClCG07G014730, ClCG08G006270, and ClCG08G012560) encoding receptor-like protein/kinases (RLPs/RLKs) were identified in this pathway. ClCG05G016550, ClCG05G017520, ClCG07G014730, and ClCG08G012560 were commonly hypermethylated for 25d_ck and hypomethylated for 25d_48h under the CHH context, ClCG08G006270 was hypomethylated for 25d_48h under the CHH context, and ClCG05G025640 was hypomethylated for 48h_ck under the CG context, hypermethylated for 25d_ck under the $\mathrm{CHH}$ context, and hypomethylated for 25d_48h under CG and CHH contexts.

\subsubsection{DMR-Associated Genes Involved in ABC Transporters}

Six DMGs were involved in the ATP-Binding Cassette (ABC) transporter pathway (ko02010) (Table S4). ClCG01G005380 encoding ABC transporter B family member 28 was hypermethylated for 25d_ck and hypomethylated for 25d_48h under the CHH context. One ABC transporter B family member 4 gene, ClCG11G013200, was hypermethylated for 48h_ck and hypomethylated for 25d_48h under the CHH context. Two DMGs (ClCG02G018950 and ClCG09G018580) encoding ABC transporter B family members 15 and 25 were hypermethylated for 48h_ck under the $\mathrm{CHH}$ context. Another two DMGs (ClCG02G007990 and ClCG10G012900), encoding ABC transporter B family members 13 and 19, were hypermethylated for 25d_ck and hypomethylated for 48h_ck and 25d_48h under the $\mathrm{CHH}$ context.

\subsection{Association between DNA Methylation and Gene Expression}

Changes in methylation levels in genomic regions tend to be associated with alteration in gene expression [34]. To analyze the expression changes of DMGs, we carried out the RNA-sequencing (RNA-seq) analysis for watermelon leaves sampled at the same stages with WGBS. Among the 
2788 DMGs, 1787 were detected and expressed (Table S5), and 362 of them showed differential expression among three libraries (Table S5 and Figure 10). These 362 DMGs were clustered into four clusters (I-IV) based on the $\log _{2}$ (FPKM) for ck, 48 hpi, and 25 dpi libraries (Figure 10a). In addition, these 362 differentially expressed DMGs were mainly divided into two clusters according to the $\log _{2}$ (FC) for 48h_ck, 25d_ck, and 25d_48h (Figure 10b). DMGs of Cluster Ishowed upregulated profiles, whereas DMGs of Cluster IIshowed downregulated profiles during the process of CGMMV infection (Figure 10b).
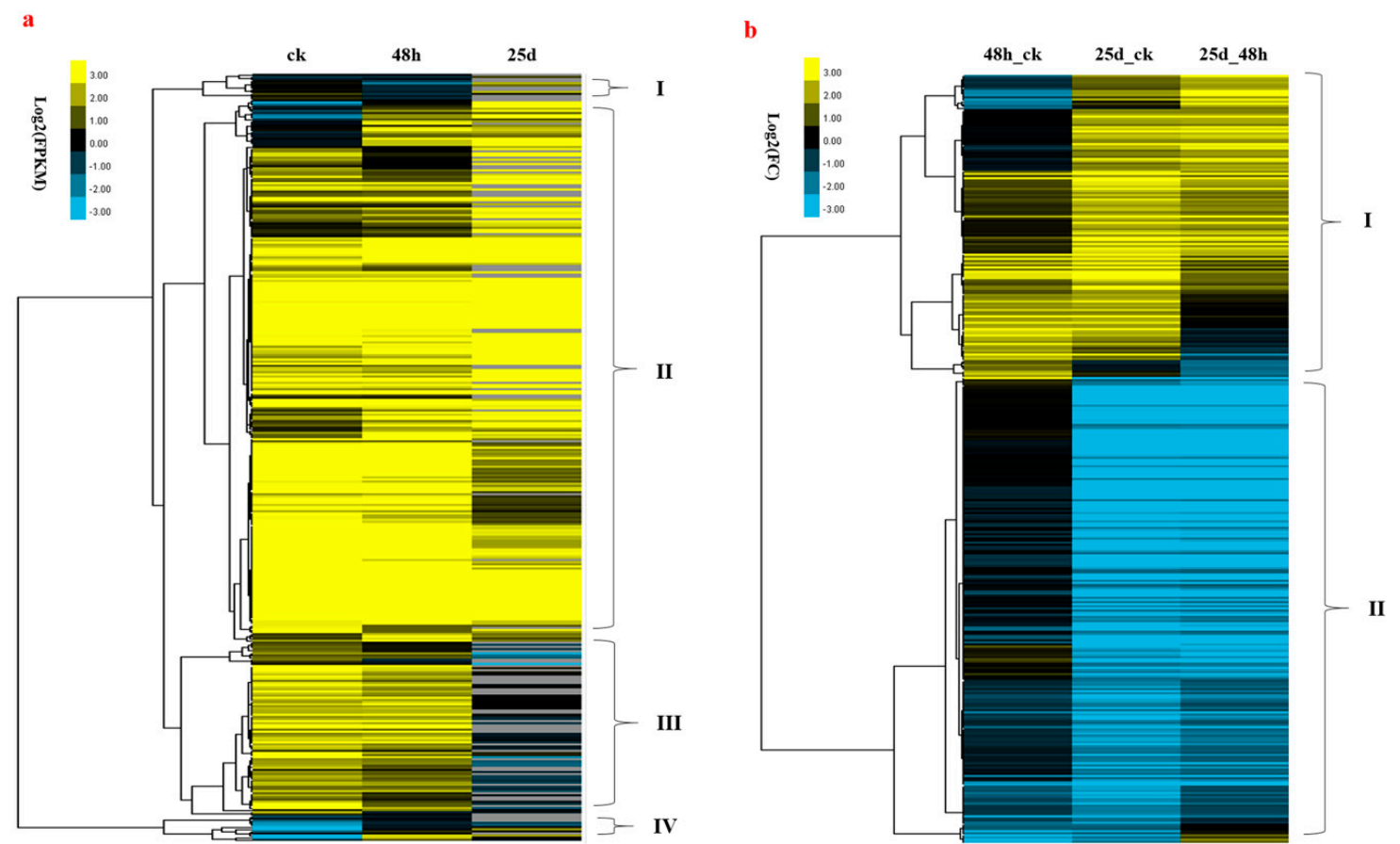

Figure 10. Hierarchical clustering of expression profiles for 362 differentially expressed DMGs among three libraries. (a) The $\log _{2}$ (Fragments Per Kilobase of exon per Million reads) for ck, 48 hpi, and 25 dpi libraries; (b) the $\log _{2}$ (FoldChange) for $48 \mathrm{~h} \_c k, 25 \mathrm{~d} \_c k$, and $25 \mathrm{~d} \_48 \mathrm{~h}$. The trees were generated using the Cluster 3.0 (https://www.encodeproject.org/software/cluster/) and Java Treeview (http://jtreeview.sourceforge.net/).

Ten of the 38 (26.31\%) DMGs under the CG context, 12 of the 47 (25.53\%) DMGs under the CHG context, and 343 of the 1720 (19.94\%) DMGs under the CHH context showed differential expression among three libraries (Table S5). The similar percentages of differentially expressed DMGs under $\mathrm{CG}, \mathrm{CHG}$, and $\mathrm{CHH}$ contexts indicated the expression changes did not correlate with the methylated sequence contexts. In detail, ten DMGs under the CG context, including ClCG01G001630 (dof zinc finger protein DOF4.6-like), ClCG01G003020 (protein P21-like), ClCG02G001570 (scarecrow-like protein 32), ClCG02G005880 (calmodulin-like protein 1), ClCG02G018820 (peroxidase 7-like), ClCG05G005770 (uncharacterized), ClCG05G006560 (psbB), ClCG10G001720 (transcriptional regulator SUPERMAN), ClCG10G013330 (photosynthetic NDH subunit of subcomplex B2), and ClCG11G013230 (uncharacterized protein) were differentially expressed during the CGMMV infection. Under the CHG context, twelve DMGs, such as ClCG01G006770 (purple acid phosphatase 17), ClCG01G006860 (hypothetical protein), ClCG01G013770 (unannotated), ClCG02G001900 (hypothetical protein), ClCG03G007120 (coatomer subunit zeta-1-like), ClCG04G002160 (anaphase-promoting complex subunit 6), ClCG04G009510 (type-1 glutamine synthetase 1-like), ClCG05G026970 (uncharacterized protein), ClCG07G017160 (glycine-rich cell wall structural protein 2), ClCG08G009690 (NADH dehydrogenase subunit 7), ClCG09G013770 (hypothetical protein), ClCG10G003690 (germacrene D synthase-like), showed differential expression. Under the CHH context, DMGs encoding cytochrome P450, chlorophyll $\mathrm{a}-\mathrm{b}$ binding protein, leucine-rich repeats (LRR) receptor-like serine/threonine-protein kinase, cellulose 
synthase, respiratory burst oxidase homolog protein, calcium-binding protein, pectinesterase, $A B C$ transporter, auxin transporter, NAC, and MYB transcription factor, were differentially expressed during the process of CGMMV infection.

Moreover, the relationship between methylation type and gene expression was analyzed. For example, the hyper-methylated ClCG01G001630 was downregulated for 48h_ck under CG context; however, under the same context, the hyper-methylated ClCG01G003020 was upregulated for 48h_ck. Under the CHG context, ClCG01G013770, ClCG02G001900, and ClCG03G007120 were hypo-methylated and the expression was commonly increased for 25d_48h, while the hypo-methylated ClCG01G006770, ClCG04G009510, and ClCG05G026970 were decreased for 25d_48h. The results indicated that the hyperor hypo-methylated genes did not have the correlation with gene upregulation or downregulation.

\subsection{Expression of Genes Invovled in the RdDM Pathway and RNA Interference in Watermelon}

RdDM is the major small RNA-mediated epigenetic pathway in plants [24]. The dynamic expression of genes in the RdDM pathway, including CMT, DRM2, RDR2, DCL3, AGO4, and AGO6, were analyzed in present study (Table 3). Three CMT genes (ClCG03G013970, ClCG10G001140 and ClCG11G000400) were detected and ClCG03G013970 was significantly down-regulated at 48h_ck and up-regulated at 25d_48h. Two genes (ClCG10G014050 and ClCG06G004300) encoding DRM2 expressed in three libraries. ClCG06G004300 showed significant upregulation at $25 \mathrm{dpi}$ compared with ck and $48 \mathrm{hpi}$, and ClCG06G004300 gradually reduced the expression during the process of CGMMV infection. ClCG06G016860 encoding RDR2 showed decreased expression during the process of CGMMV infection. Three genes (ClCG08G009740, ClCG10G005250, and ClCG02G002110) encoding DCL3 were detected. Among which, ClCG08G009740 was slightly downregulated during the process of CGMMV infection, and ClCG10G005250 and ClCG02G002110 were slightly upregulated at $48 \mathrm{hpi}$, then downregulated at 25 dpi compared with ck. Moreover, AGO4 (ClCG02G024220) and AGO6 (ClCG09G011170) was significantly downregulated at 25 dpi compared with ck. Generally, genes involved in the RdDM pathway showed downregulation after the CGMMV infection, especially at the 25 dpi stage.

Table 3. Expression of genes involved in the RdDM pathway and RNA interference in watermelon.

\begin{tabular}{|c|c|c|c|c|c|c|c|}
\hline Genes & Annotation & FPKM_ck & FPKM_48h & FPKM $\_25 d$ & $\log _{2}\left(48 h \_c k\right)$ & $\log _{2}\left(25 d \_c k\right)$ & $\log _{2}\left(25 d \_48 h\right)$ \\
\hline ClCG08G009740 & DCL3 & 6.63 & 5.95 & 5.49 & -0.16 (down) & -0.27 (down) & -0.11 (down) \\
\hline ClCG10G005250 & DCL3 & 4.08 & 5.28 & 2.96 & 0.37 (up) & -0.46 (down) & -0.84 (down) \\
\hline ClCG02G002110 & DCL3 & 1.49 & 1.83 & 0.00 & 0.30 (up) & Inf (down) & Inf (down) \\
\hline ClCG06G012100 & DCL4 & 2.37 & 2.63 & 1.78 & 0.15 (up) & -0.41 (down) & -0.56 (down) \\
\hline ClCG03G010530 & DCL2 & 5.64 & 5.38 & 21.89 & -0.07 (down) & 1.96 (up) & 2.03 (up) \\
\hline ClCG02G024220 & AGO4 & 44.49 & 38.07 & 20.30 & -0.23 (down) & -1.13 (down) & -0.91 (down) \\
\hline ClCG09G011170 & AGO6 & 1.40 & 0.70 & 0.00 & -0.99 (down) & Inf (down) & Inf (down) \\
\hline ClCG00G004530 & AGO10 & 1.71 & 0.86 & 0.80 & -1.00 (down) & -1.10 (down) & -0.10 (down) \\
\hline ClCG04G012520 & AGO1B & 40.68 & 34.46 & 49.82 & -0.24 (down) & 0.29 (up) & 0.53 (up) \\
\hline ClCG05G023990 & AGO & 9.93 & 6.31 & 6.98 & -0.65 (down) & -0.51 (down) & 0.15 (up) \\
\hline ClCG05G004800 & AGO & 40.68 & 34.46 & 49.82 & -0.24 (down) & 0.29 (up) & 0.53 (up) \\
\hline ClCG01G014010 & AGO & 5.19 & 6.18 & 6.52 & 0.25 (up) & 0.33 (up) & 0.08 (up) \\
\hline ClCG03G013970 & CMT & 6.68 & 2.12 & 5.87 & -1.65 (down) & -0.19 (down) & 1.47 (up) \\
\hline ClCG10G001140 & CMT & 15.47 & 17.87 & 14.80 & 0.21 (up) & -0.06 (down) & -0.27 (down) \\
\hline ClCG11G000400 & CMT & 6.53 & 6.09 & 3.37 & -0.10 (down) & -0.96 (down) & -0.85 (down) \\
\hline ClCG10G014050 & DRM2 & 20.84 & 19.90 & 14.12 & -0.07 (down) & -0.56 (down) & -0.49 (down) \\
\hline ClCG06G004300 & DRM2 & 8.24 & 8.25 & 17.88 & 0.00 & 1.12 (up) & 1.12 (up) \\
\hline ClCG01G006600 & RDR & 0.06 & 0.41 & 0.00 & 2.76 (up) & Inf (down) & Inf (down) \\
\hline ClCG01G006450 & RDR & 0.28 & 0.02 & 1.04 & -4.04 (down) & 1.86 (up) & 5.90 (up) \\
\hline ClCG08G013320 & RDR6 & 7.14 & 4.69 & 1.40 & -0.61 (down) & -2.35 (down) & -1.74 (down) \\
\hline ClCG07G005340 & RDR1a & 0.86 & 1.12 & 1.18 & 0.38 (up) & 0.46 (up) & 0.07 (up) \\
\hline ClCG06G016860 & RDR2 & 3.61 & 2.93 & 1.83 & -0.30 (down) & -0.98 (down) & -0.68 (down) \\
\hline
\end{tabular}

FPKM: Fragments per kilobase of exon per million reads.

In addition, we also analyzed the expression of other genes encoding DCLs, RDRs, and AGOs that direct the antiviral RNAi defense in watermelon. DCL2 (ClCG03G010530) was significantly upregulated at $25 \mathrm{dpi}$ compared with ck and 48 hpi. Expression of DCL4 (ClCG06G012100) slightly increased at $48 \mathrm{hpi}$, then decreased at 25 dpi compared with ck. Five genes (ClCG00G004530, ClCG04G012520, ClCG05G023990, ClCG05G004800 and ClCG01G014010) encoding AGO proteins were detected and expressed. ClCG00G004530 (AGO10) was gradually downregulated and ClCG01G014010 (AGO) was 
gradually upregulated in the process of CGMMV infection. ClCG04G012520 (AGO1B), ClCG05G023990 (AGO), and ClCG05G004800 (AGO) commonly decreased at $48 \mathrm{hpi}$, and then increased at $25 \mathrm{dpi}$. Four genes (ClCG01G006600, ClCG01G006450, ClCG08G013320 and ClCG07G005340) encoding RDRs were expressed. Among them, ClCG08G013320 (RDR6) significantly reduced the expression after infection with CGMMV.

\section{Discussion}

DNA methylation is a common feature of eukaryotic epigenomes. Many studies have improved our understanding of the variation in DNA methylation associated with the defense response to viral infection in plants, including beet severe curly top virus in Arabidopsis [54], TYLCV in tomato [39], and CMV in tobacco [34]. In this study, we tested the effect of global DNA methylation on CGMMV invasion in watermelon for the first time and determined its role during CGMMV infection.

\subsection{Global DNA Methylation Level and Genomic Distribution of DNA Methylation under Cucumber Green} Mottle Mosaic Virus Infection

Global DNA methylation levels varied with different stages of the CGMMV infection, being highest in the ck library and lowest in the $48 \mathrm{~h}$ library (Figure 2). Additionally, the methylation density was highest under the CHH context, followed by CHG and CG contexts (Figure 2). This is consistent with a previous finding that methylated regions were enriched with $\mathrm{CHH}$ sequence contexts in response to CMV infection in tobacco [34]. In the present study, methylation levels were highest for the up-2k genomic region under $\mathrm{CHG}$ and $\mathrm{CHH}$ contexts (Figure 6). DNA methylation of promoters (up-2k) would lead to changes in gene expression by altering the chromatin structure and blocking transcriptional initiation [26]. However, under the CG context, the highest methylation level was observed within introns, followed by up-2k, down-2k, gene body, cds, utl3, and utl5 in all three libraries (Figure 6). DNA methylation of gene bodies is positively correlated with transcriptional activation and plays an important role in silencing repetitive elements and alternative splicing $[25,26]$.

\subsection{DMRs and DMGs Mainly Gathered under the CHH Context During Cucumber Green Mottle Mosaic Virus Infection}

The number of DMRs increased from 16,483 to 30,523 during the CGMMV infection, and hyper DMRs were highest in 25d_ck and lowest in 25d_48h, while hypo DMRs were highest in 25d_48h and lowest in 25d_ck (Figure 7). A total of 2788 DMGs were screened out of the three libraries, with 863, 1520 , and 1691 detected for 48h_ck, 25d_ck, and 25d_48h, respectively (Table S2). Of note, the vast majority of DMRs and DMGs occurred under the CHH context, implying that DNA methylation in this state plays an important role in the response to CGMMV infection in watermelon.

\subsection{DMR-Associated Genes Enriched in Plant-Pathogen Interactions During Cucumber Green Mottle Mosaic Virus Infection}

Within the plant-pathogen interaction, plants have evolved innate immune systems that recognize the presence of pathogens and initiate effective defense responses, whereas pathogens have evolved effector proteins that suppress host immune responses [55]. We found that DMGs encoding CaM-like protein, calcium-binding protein, WRKYs, PR1a, RBOHs, CNGCs, RIN4, and BAK1 were involved in plant-pathogen interaction pathways, and most of these genes were hypermethylated for 25d_ck and hypomethylated for 25d_48h (Table S4).

The $\mathrm{Ca}^{2+}$ signaling pathway plays an important role in plant defense to biotic and abiotic stresses [56]. In the present study, two DMGs (ClCG03G008520 and ClCG05G003310) encoding calcium-binding proteins CML22 and CML31 were hypomethylated under the CHH context during the CGMMV infection, while DMGs ClCG02G005880 and ClCG02G016580, encoding CaM-like protein 1 and CaM-like protein 8, were hypomethylated under CG and CHH contexts, respectively. CaM is the primary cellular $\mathrm{Ca}^{2+}$ receptor which recognizes and binds specific $\mathrm{Ca}^{2+}$ signals through the 
EF-hand domain and interacts with the calmodulin-binding domains of target calmodulin-binding proteins to transfer $\mathrm{Ca}^{2+}$ signals [57]. Many WRKY TFs have been demonstrated to associate with various defense responses in plants [58-60]. For example, OsWRKY67 induces the transcription of a range of defense-related genes, including those involved in the salicylic acid-dependent pathway [58]. Moreover, OsWRKY62 negatively regulates the basal and Xa21-mediated resistance to bacterial blight [59], and OsWRKY28 is negatively correlated with blast disease resistance [60]. In this study, three DMGs (ClCG02G002690, ClCG02G019200, and ClCG07G007870) encoding WRKYs were identified. ClCG02G002690 (WRKY1) was hypermethylated for 25d_ck and hypomethylated for 25d_48h under the CHH context, while ClCG02G019200 (WRKY26) was hypermethylated for 25d_ck and ClCG07G007870 (WRKY22) was hypomethylated for 48h_ck under the CHH context. These WRKYs might regulate the watermelon response to CGMMV infection.

The PRs are associated with the development of systemic acquired resistance against further infection enforced by fungi, bacteria, and viruses [61]. PR1a was the first PR-1 member to be purified and characterized [62], and PR1a-overexpressing plants exhibited increased tolerance to the oomycete pathogens Phytophthora parasitica var. nicotianae and Peronospora tabacina [63]. In this study, ClCG02G007230 encoding PR1a was hypomethylated for 25d_48h under the CHH context, suggesting ClCG02G007230 is associated with the CGMMV response in watermelon.

The $\mathrm{RBOHs}$ have been established as important second messengers that regulate the expression of hundreds of genes in response to stresses [64,65]. Three DMGs (ClCG01G002290, ClCG03G017090, and ClCG07G007770) encoding RBOHs were identified in this pathway in the present study, and all were hypomethylated for 25d_48h under the CHH context. Arabidopsis CNGCs were previously shown to be involved in plant defense responses against diseases, with AtCNGC2 and AtCNGC4 mutants (dnd1 and dnd2) demonstrating similar phenotypes such as impaired hypersensitive reactions and enhanced broad-spectrum resistance to bacterial pathogens [66,67]. Five DMGs (ClCG04G009060, ClCG05G008780, ClCG05G008800, ClCG06G016900, and ClCG10G014650) encoding CNGCs were detected in this pathway in the present study, and all were methylated under the CHH context.

RIN4 is the only known protein that regulates both branches of the plant immune system. After pathogen-associated molecular pattern (PAMP) treatment, RIN4 overexpression lines exhibited decreased callose deposition and enhanced growth of virulent and type III secretion-deficient Pst, indicating a reduction in pattern-triggered immunity [68]. Moreover, RIN4 associates with the C-terminal autoinhibitory domain of $\mathrm{PM} \mathrm{H}^{+}$-ATPase to regulate the response of leaf stomata to PAMPs [69]. A RIN4 gene (ClCG05G003210) was hypermethylated for 25d_ck and hypomethylated for $25 \mathrm{~d} \_48 \mathrm{~h}$ under the $\mathrm{CHH}$ context in the present study. BAK1 is an essential co-receptor of multiple receptor complexes, with well-characterized roles in the regulation of immunity and defense-related programmed cell death [70,71]. Interestingly, DMG ClCG09G015110 encoding BAK1 was hypermethylated for $48 \mathrm{~h} \_c k$ under the $\mathrm{CHH}$ context in the present study, suggesting it functions in the early response to CGMMV infection in watermelon.

\subsection{DMR-Associated Genes Enriched in Toll-Like Receptor Signaling During Cucumber Green Mottle Mosaic Virus Infection}

Toll-like receptor signaling plays a central role in the immune response by recognizing pathogen-associated molecular patterns from bacteria and viruses [72]. Serine/threonine protein kinases (STKs) are receptor proteins that mediate signal transduction in plant defense. Serine/threonine protein kinases are mainly involved in the recognition and transduction of pathogen signals during the interaction of plants and microbes [73,74]. Four DMGs (ClCG02G020720, ClCG07G002710, ClCG09G010710, and ClCG11G011240) encoding STKs were identified in the Toll-like receptor signaling pathway in the present study. ClCG07G002710 was hypomethylated for 48h_ck under the CG context and the other three DMGs were methylated under the $\mathrm{CHH}$ context. Receptor-like kinases/proteins (RLKs/RLPs) perceive and initiate PAMP-triggered immunity, which is the first layer of plant innate immunity [75]. In this study, six DMGs encoding RLKs/RLPs were identified. Except for ClCG05G025640 
(RLK3), which was hypomethylated for $48 \mathrm{~h} \_c k$ under the CG context, hypermethylated for 25d_ck under the $\mathrm{CHH}$ context, and hypomethylated for $25 \mathrm{~d} \_48 \mathrm{~h}$ under CG and CHH contexts, the remaining DMGs were commonly methylated under the CHH context. These STKs and RLKs/RLPs play an important role in activating plant innate immunity.

\subsection{DMR-Associated Genes Enriched in ABC Transporters During Cucumber Green Mottle Mosaic Virus Infection}

The $A B C$ transporter is required to resist cell wall penetration and subsequent haustorium formation by the non-host barley powdery mildew pathogen Blumeria graminis $\mathrm{f}$. sp. hordei $(B g h)$ and contributes to resistance to many other fungal and oomycete pathogens [76]. Six DMGs (ClCG01G005380, ClCG02G007990, ClCG02G018950, ClCG09G018580, ClCG10G012900, and ClCG11G013200) encoding $\mathrm{ABC}$ transporters were detected in the present study (Table S4). Except for ClCG10G012900, which was hypomethylated for $25 \mathrm{~d} \_48 \mathrm{~h}$ under the CHG context, the remaining DMGs were methylated under the $\mathrm{CHH}$ context. These DMGs might contribute to resistance to CGMMV infection in watermelon.

\subsection{Relationship between DNA Methylation and Gene Expression}

Plant DNA methylation is interpreted by cells to maintain transposon silencing, promote chromatin structure, and ensure the normal expression of certain genes [20]. In Arabidopsis, correlations of methylation and expression differed between gene body methylation and TE methylation, gene body methylation is usually positively correlated and TE methylation is usually negatively correlated with expression [77]. In soybean, no obvious correlation between methylation changes and transcriptional variation of genes was detected based on transcriptional profiling [78]. In the present study, only 362 of the 2788 DMGs showed different expression levels in response to CGMMV infection (Table S5 and Figure 10). These differentially expressed DMGs were detected under the CG, CHG, and CHH contexts and the relationship between methylation changes and gene expression was unclear. In plants, only the methylation of gene bodies in the mCG context has been associated with higher gene expression, and more specifically, constitutive gene expression [26]. These methylated genes were characterized by the absence of $\mathrm{mCG}$ in and around the transcriptional start site and transcriptional termination site [79]. The methylation of gene regulatory elements also affects TF binding and gene expression. For example, in maize, higher $\mathrm{mCHH}$ levels upstream and downstream of genes have been associated with high gene expression [80]. Taken together, these findings suggest that much remains to be explored about DNA methylation and its impact on the regulation of gene expression.

\subsection{RNA Interference and RdDM Pathways Associated with Watermelon Antiviral Processes}

One of the first pieces of evidence for epigenomic regulation of plant immunity appeared with the description of the control of viral virulence through RNA silencing [81]. In tobacco, $\mathrm{CHH}$ hypomethylation was dominant at 16-day post-CMV infection, together with the loss of 24-nt siRNA distribution across the gene body [34]. In Arabidopsis, CMV infection with $2 \mathrm{~b}$ deletion induced an enhanced population of 21-nt siRNAs and decreased the proportion of 24-nt siRNAs [82]. Rice stripe virus (RSV)-infected rice leaves revealed that siRNAs were derived almost equally from virion and the complementary RNA strands, and the length of siRNAs were mostly 20-22 nt long [83]. In our previous study, CGMMV infection induced the 21- and 22-nt siRNAs and reduced the level of 24-nt siRNAs in watermelon leaves [18]. Upon infection with the biotrophic fungal pathogen Bgt, AGO4a was significantly downregulated, a response similar to that in Arabidopsis plants elicited by the bacterial PAMP flg22 [37]. In this study, AGO4 was also significantly downregulated during the process of CGMMV infection in watermelon (Table 3), which was accompanied by a substantial reduction in AGO4-sorted 24-nt siRNA levels as our previous study indicated [18].

DNA methylation mediated by 21-nt siRNAs may represent a different mechanism from the RdDM pathway, in which 24-nt siRNAs play a major role [84]. CHH methylation is usually mediated by RdDM pathway targeted in the short TEs and the edges of TEs [35]. In present study, $\mathrm{CHH}$ 
methylation occurs in most DMGs, suggesting the RdDM-directed $\mathrm{CHH}$ methylation plays important roles in watermelon response to the CGMMV. Genes encoding CMT, DRM2, RDR2, DCL3, AGO4, and AGO6 involved in the RdDM pathway were mostly downregulated after the CGMMV infection in the present study, which suggests depression of the RdDM pathway would initiate the immune response in watermelon. This observation was consistent with reduced DNA methylation, which promotes host defense responses against pathogen infection in wheat and Arabidopsis [35,83]. Thus, our results indicate reduced RdDM-directed DNA methylation of CHH and increased 21- and 22-nt siRNAs acting a synergetic effect on the antiviral process in watermelon.

\section{Conclusions}

DNA methylation plays important roles in plant response to viral infection. In the present study, whole-genome bisulfite sequencing of watermelon leaves sampled at ck, 48 hpi, and 25 dpi were carried out. Methylation level at $48 \mathrm{hpi}$ was lower than at ck and at $25 \mathrm{dpi}$. Most DMRs and DMGs were gathered under the $\mathrm{CHH}$ context during the process of CGMMV infection. DMGs involving in pathways of secondary biosynthesis and metabolism, plant-pathogen interactions, Toll-like receptor signaling, and ABC transporters were significantly enriched. Moreover, a range of DMGs encoding defense-related proteins were obtained. Correlation of DNA methylation and gene expression was analyzed by RNA-seq and no clear relationship was detected. Most genes involved in the RdDM pathway reduced their expression during the process of CGMMV infection. This study provides the DNA methylation variation in response to viral infection in watermelon for the first time. In addition, these results indicate the reduced RdDM-directed $\mathrm{CHH}$ methylation and increased 21- and 22-nt siRNAs act as a synergetic effect on antiviral defense in watermelon.

Supplementary Materials: The following are available online at http://www.mdpi.com/2073-4425/10/5/344/s1, Table S1: Differentially methylated regions (DMRs) identified in watermelon for 48h_ck, 25d_ck, and 25d_48h under CG, CHG, and CHH contexts, Table S2: Differentially methylated genes (DMGs) and their annotation identified in watermelon for $48 \mathrm{~h} \_c k, 25 \mathrm{~d} \_c k$, and 25d_48h under CG, CHG, and CHH contexts, Table S3: KEGG pathway enrichment of DMGs for 48h_ck, 25d_ck and 25d_48h, Table S4: DMGs associated with CGMMV response in watermelon, Table S5: Expression of DMGs in response to CGMMV infection by RNA-Seq, Figure S1: The dynamics of genic DNA methylation for 15 selected CGMMV response-related DMGs using the IGV genome browser.

Author Contributions: Y.S. and M.F. conceived and designed the experiments; Y.S. carried out the bioinformatics analysis and wrote the manuscript; M.F. and Y.H. gave insightful suggestions.

Funding: This work was funded by the Zhejiang Provincial Natural Science Foundation of China (LQ18C150002), the Key Laboratory of Biology and Genetic Improvement of Horticultural Crops, Ministry of Agriculture, P.R. China (IVF201801), the National Natural Science Foundation of China (31572145), and the Zhejiang Major Science and Technology Project for New Vegetable Variety Breeding (2016C02051-4-2).

Acknowledgments: We thank Sarah Williams from Liwen Bianji, Edanz Group China (www.liwenbianji.cn), for editing the English text of a draft of this manuscript.

Conflicts of Interest: The authors declare no conflict of interest. The funders had no role in the design of the study; in the collection, analyses, or interpretation of data; in the writing of the manuscript, or in the decision to publish the results.

\section{References}

1. Ainsworth, G.C. Mosaic disease of the cucumber. Ann. Appl. Biol. 1935, 22, 55-67. [CrossRef]

2. Inoue, T.; Inoue, N.; Asatani, M.; Mitsuhata, K. Studies on cucumber green mottle mosaic virus in Japan. Nogaku Kenkyu 1967, 51, 175-186.

3. Komuro, Y. Cucumber green mottle mosaic virus on cucumber and watermelon and melon necrotic spot virus on muskmelon. Jpn. Agric. Res. Q. 1971, 6, 41-45.

4. Vasudeva, R.S.; Raychaudhuri, S.P.; Singh, J. A new strain of Cucumis virus 2. Ind. Phytopathol. 1949, 2, 180-185.

5. Motoyoshi, F.; Nishiguchi, M. Control of virus diseases by attenuated virus strains, comparison between attenuated strains of cucumber green mottle mosaic virus and tobacco mosaic virus. Gamma Field Symp. 1988, 27, 91-109. 
6. Dombrovsky, A.; Tran-Nguyen, L.T.T.; Jones, R.A.C. Cucumber green mottle mosaic virus: Rapidly increasing global distribution, etiology, epidemiology and management. Ann. Rev. Phytopathol. 2017, 55, 231-256. [CrossRef] [PubMed]

7. Lee, S.Y.; Win, N.K.K.; Cho, D.M.; Lee, S.H.; Jung, H.Y. Cucumber green mottle mosaic virus (CGMMV) can induce hair-like tissues on genus Cucumis seeds. Sci. Hortic. 2012, 146, 76-80. [CrossRef]

8. Li, R.; Zheng, Y.; Fei, Z.; Ling, K.S. First complete genome sequence of an emerging cucumber green mottle mosaic virus isolate in North America. Genome Announc. 2015, 3, e00452-15. [CrossRef]

9. Tesoriero, L.A.; Chambers, G.; Srivastava, M.; Smith, S.; Conde, B.; Tran-Nguyen, L.T.T. First report of cucumber green mottle mosaic virus in Australia. Australas. Plant Dis. Notes 2016, 11, 1. [CrossRef]

10. Pop, I.; Jilaveanu, A. Identification of cucumber green mottle virus in Romania. Analele Institutului de Cercetari Pentru Protectia Plantelor 1985, 18, 43-47.

11. Varveri, C.; Vassilakos, N.; Bem, F. Characterization and detection of Cucumber green mottle mosaic virus in Greece. Phytoparasitica 2002, 30, 493-501. [CrossRef]

12. Li, X.; An, M.; Xia, Z.; Bai, X.; Wu, Y. Transcriptome analysis of watermelon (Citrullus lanatus) fruits in response to Cucumber green mottle mosaic virus (CGMMV) infection. Sci. Rep. 2017, 7, 16747. [CrossRef]

13. Sun, Y.; Fan, M.; He, Y. Transcriptome analysis of watermelon leaves reveals candidate genes responsive to Cucumber green mottle mosaic virus infection. Int. J. Mol. Sci. 2019, 20, 610. [CrossRef] [PubMed]

14. Liu, H.W.; Luo, L.X.; Liang, C.Q.; Jiang, N.; Liu, P.F.; Li, J.Q. High-throughput sequencing identifies novel and conserved cucumber (Cucumis sativus L.) microRNAs in response to Cucumber green mottle mosaic virus infection. PLoS ONE 2015, 10, e0129002. [CrossRef] [PubMed]

15. Sun, Y.; Niu, X.; Fan, M. Genome-wide identification of cucumber green mottle mosaic virus-responsive microRNAs in watermelon. Arch. Virol. 2017, 162, 2591-2602. [CrossRef] [PubMed]

16. Li, Y.; Deng, C.; Shang, Q.; Zhao, X.; Liu, X.; Zhou, Q. Characterization of siRNAs derived from cucumber green mottle mosaic virus in infected cucumber plants. Arch. Virol. 2016, 161, 455-458. [CrossRef] [PubMed]

17. Li, J.; Zheng, H.; Zhang, C.; Han, K.; Wang, S.; Peng, J.; Lu, Y.; Zhao, J.; Xu, P.; Wu, X.; Li, G.; Chen, J.; Yan, F. Different virus-derived siRNAs profiles between leaves and fruits in Cucumber green mottle mosaic virus-infected Lagenaria siceraria plants. Front. Microbiol. 2016, 7, 1797. [CrossRef] [PubMed]

18. Sun, Y.; Niu, X.; Cui, D.; Fan, M. High-throughput sequencing reveals vsiRNAs derived from Cucumber green mottle mosaic virus-infected watermelon. Horticul. Plant J. 2017, 3, 17-22. [CrossRef]

19. Kim, M.Y.; Zilberman, D. DNA methylation as a system of plant genomic immunity. Trends Plant Sci. 2014, 19, 320-326. [CrossRef]

20. Niederhuth, C.E.; Schmitz, R.J. Putting DNA methylation in context: From genomes to gene expression in plants. Biochim. Biophys. Acta 2017, 1860, 149-156. [CrossRef]

21. Cokus, S.J.; Feng, S.; Zhang, X.; Chen, Z.; Merriman, B.; Haudenschild, C.D.; Pradhan, S.; Nelson, S.F.; Pellegrini, M.; Jacobsen, S.E. Shotgun bisulfite sequencing of the Arabidopsis genome reveals DNA methylation patterning. Nature 2008, 452, 215-219. [CrossRef]

22. Cao, X.; Jacobsen, S.E. Locus-specific control of asymmetric and CpNpG methylation by the DRM and CMT3 methyltransferase genes. Proc. Natl. Acad. Sci. USA 2002, 99, 16491-16498. [CrossRef] [PubMed]

23. Stroud, H.; Do, T.; Du, J.; Zhong, X.; Feng, S.; Johnson, L.; Patel, D.J.; Jacobsen, S.E. Non-CG methylation patterns shape the epigenetic landscape in Arabidopsis. Nat. Struct. Mol. Boil. 2014, 21, 64-72. [CrossRef]

24. Matzke, M.A.; Mosher, R.A. RNA-directed DNA methylation: An epigenetic pathway of increasing complexity. Nat. Rev. Genet. 2014, 15, 394-408. [CrossRef]

25. Jones, P.A. Functions of DNA methylation: Islands, start sites, gene bodies and beyond. Nat. Rev. Genet. 2012, 13, 484-492. [CrossRef]

26. Bewick, A.J.; Schmitz, R.J. Gene body DNA methylation in plants. Curr. Opin. Plant Biol. 2017, 36, $103-110$. [CrossRef] [PubMed]

27. Finnegan, E.J.; Peacock, W.J.; Dennis, E.S. Reduced DNA methylation in Arabidopsis results in abnormal plant development. Proc. Natl. Acad. Sci. USA 1996, 93, 8449-8454. [CrossRef]

28. Xiao, W.; Custard, K.D.; Brown, R.C.; Lemmon, B.E.; Harada, J.J.; Goldberg, R.B.; Fischer, R.L. DNA methylation is critical for Arabidopsis embryogenesis and seed viability. Plant Cell 2006, 18, 805-814. [CrossRef] [PubMed]

29. Jacobsen, S.E.; Sakai, H.; Finnegan, E.J.; Cao, X.; Meyerowitz, E.M. Ectopic hypermethylation of flower-specific genes in Arabidopsis. Curr. Biol. 2000, 10, 179-186. [CrossRef] 
30. Kim, M.; Ohr, H.; Lee, J.W.; Hyun, Y.; Fischer, R.L.; Choi, Y. Temporal and spatial downregulation of Arabidopsis MET1 activity results in global DNA hypomethylation and developmental defects. Mol. Cells 2008, 26, 611-615.

31. Xiao, W.; Brown, R.C.; Lemmon, B.E.; Harada, J.J.; Goldberg, R.B.; Fischer, R.L. Regulation of seed size by hypomethylation of maternal and paternal genomes. Plant Physiol. 2006, 142, 1160-1168. [CrossRef] [PubMed]

32. Hossain, M.S.; Kawakatsu, T.; Kim, K.D.; Zhang, N.; Nguyen, C.T.; Khan, S.M.; Batek, J.M.; Joshi, T.; Schmutz, J.; Grimwood, J.; Schmitz, R.J.; Xu, D.; Jackson, S.A.; Ecker, J.R.; Stacey, G. Divergent cytosine DNA methylation patterns in single-cell, soybean root hairs. New Phytol. 2017, 214, 808-819. [CrossRef] [PubMed]

33. Ganguly, D.R.; Crisp, P.A.; Eichten, S.R.; Pogson, B.J. The Arabidopsis DNA methylome is stable under transgenerational drought stress. Plant Physiol. 2017, 175, 1893-1912. [CrossRef] [PubMed]

34. Wang, C.; Wang, C.; Xu, W.; Zou, J.; Qiu, Y.; Kong, J.; Yang, Y.; Zhang, B.; Zhu, S. Epigenetic changes in the regulation of Nicotiana tabacum response to Cucumber mosaic virus infection and symptom recovery through single-base resolution methylomes. Viruses 2018, 10, 402. [CrossRef] [PubMed]

35. Geng, S.; Kong, X.; Song, G.; Jia, M.; Guan, J.; Wang, F.; Qin, Z.; Wu, L.; Lan, X.; Li, A.; Mao, L. DNA methylation dynamics during the interaction of wheat progenitor Aegilops tauschii with the obligate biotrophic fungus Blumeria graminis f. sp. tritici. New Phytol. 2019, 221, 1023-1035. [CrossRef]

36. Le, T.N.; Schumann, U.; Smith, N.A.; Tiwari, S.; Au, P.C.; Zhu, Q.H.; Taylor, J.M.; Kazan, K.; Llewellyn, D.J.; Zhang, R.; Dennis, E.S.; Wang, M. DNA demethylases target promoter transposable elements to positively regulate stress responsive genes in Arabidopsis. Genome Biol. 2014, 15, 458. [CrossRef] [PubMed]

37. Yu, A.; Lepere, G.; Jay, F.; Wang, J.; Bapaume, L.; Wang, Y.; Abraham, A.L.; Penterman, J.; Fischer, R.L.; Voinnet, O.; Navarro, L. Dynamics and biological relevance of DNA demethylation in Arabidopsis antibacterial defense. Proc. Natl. Acad. Sci. USA 2013, 110, 2389-2394. [CrossRef]

38. Wang, B.; Yang, X.; Wang, Y.; Xie, Y.; Zhou, X. Tomato yellow leaf curl virus V2 interacts with host histone deacetylase 6 to suppress methylation-mediated transcriptional gene silencing in plants. J. Virol. 2018, 92, e00036-18. [CrossRef]

39. Butterbach, P.; Verlaan, M.G.; Dullemans, A.; Lohuis, D.; Visser, R.G.; Bai, Y.; Kormelink, R. Tomato yellow leaf curl virus resistance by Ty-1 involves increased cytosine methylation of viral genomes and is compromised by cucumber mosaic virus infection. Proc. Natl. Acad. Sci. USA 2014, 111, 12942-12947. [CrossRef] [PubMed]

40. Ghoshal, B.; Sanfaçon, H. Symptom recovery in virus-infected plants: Revisiting the role of RNA silencing mechanisms. Virology 2015, 479, 167-179. [CrossRef]

41. Ding, S.W. RNA-based antiviral immunity. Nat. Rev. Immunol. 2010, 10, 632-644. [CrossRef] [PubMed]

42. Duan, C.G.; Fang, Y.Y.; Zhou, B.J.; Zhao, J.H.; Hou, W.N.; Zhu, H.; Ding, S.W.; Guo, H.S. Suppression of Arabidopsis ARGONAUTE1-mediated slicing, transgene-induced RNA silencing, and DNA methylation by distinct domains of the Cucumber mosaic virus $2 \mathrm{~b}$ protein. Plant Cell 2012, 24, 259-274. [CrossRef] [PubMed]

43. Piedra-Aguilera, A.; Jiao, C.; Luna, A.P.; Villanueva, F.; Dabad, M.; Esteve-Codina, A.; Díaz-Pendón, J.A.; Fei, Z.; Bejarano, E.R.; Castillo, A.G. Integrated single-base resolution maps of transcriptome, sRNAome and methylome of Tomato yellow leaf curl virus (TYLCV) in tomato. Sci. Rep. 2019, 9, 2863. [CrossRef] [PubMed]

44. Li, Q.; Hermanson, P.J.; Springer, N.M. Detection of DNA methylation by whole-genome bisulfite sequencing. Methods Mol. Biol. 2018, 1676, 185-196. [PubMed]

45. Wang, Z.; Wu, X.; Wu, Z.; An, H.; Yi, B.; Wen, J.; Ma, C.; Shen, J.; Fu, T.; Tu, J. Genome-Wide DNA methylation comparison between Brassica napus genic male sterile line and restorer line. Int J. Mol. Sci. 2018, 10, 19. [CrossRef] [PubMed]

46. Liang, L.; Chang, Y.; Lu, J.; Wu, X.; Liu, Q.; Zhang, W.; Su, X.; Zhang, B. Global methylomic and transcriptomic analyses reveal the broad participation of DNA methylation in daily gene expression regulation of Populus trichocarpa. Front. Plant Sci. 2019, 10, 243. [CrossRef] [PubMed]

47. Ding, C.J.; Liang, L.X.; Diao, S.; Su, X.H.; Zhang, B.Y. Genome-wide analysis of day/night DNA methylation differences in Populus nigra. PLoS ONE 2018, 13, e0190299. [CrossRef]

48. Xi, Y.; Li, W. BSMAP: Whole genome bisulfite sequence MAPping program. BMC Biol. 2009, 10, 232. [CrossRef] [PubMed]

49. Zhong, S.; Fei, Z.; Chen, Y.R.; Zheng, Y.; Huang, M.; Vrebalov, J.; McQuinn, R.; Gapper, N.; Liu, B.; Xiang, J.; Shao, Y.; Giovannoni, J.J. Single-base resolution methylomes of tomato fruit development reveal epigenome modifications associated with ripening. Nat. Biotechnol. 2013, 31, 154-159. [CrossRef] [PubMed] 
50. $\quad$ Lister, R.; Pelizzola, M.; Dowen, R.H.; Hawkins, R.D.; Hon, G.; Tonti-Filippini, J.; Nery, J.R.; Lee, L.; Ye, Z.; Ngo, Q.M.; et al. Human DNA methylomes at base resolution show widespread epigenomic differences. Nature 2009, 462, 315-322. [CrossRef]

51. Kanehisa, M.; Goto, S. KEGG: Kyoto encyclopedia of genes and genomes. Nucleic Acids Res. 2000, 28, 27-30. [CrossRef] [PubMed]

52. Thorvaldsdóttir, H.; Robinson, J.T.; Mesirov, J.P. Integrative Genomics Viewer (IGV): High-performance genomics data visualization and exploration. Brief Bioinf. 2013, 14, 178-192. [CrossRef] [PubMed]

53. Trapnell, C.; Williams, B.A.; Pertea, G.; Mortazavi, A.; Kwan, G.; van Baren, M.J.; Salzberg, S.L.; Wold, B.J.; Pachter, L. Transcript assembly and quantification by RNA-seq reveals unannotated transcripts and isoform switching during cell differentiation. Nat. Biotechnol. 2010, 28, 511-515. [CrossRef] [PubMed]

54. Yang, L.P.; Fang, Y.Y.; An, C.P.; Dong, L.; Zhang, Z.H.; Chen, H.; Xie, Q.; Guo, H.S. C2-mediated decrease in DNA methylation, accumulation of siRNAs, and increase in expression for genes involved in defense pathways in plants infected with beet severe curly top virus. Plant J. 2013, 73, 910-917. [CrossRef] [PubMed]

55. Dodds, P.N.; Rathjen, J.P. Plant immunity: Towards an integrated view of plant-pathogen interactions. Nat. Rev. Genet. 2010, 11, 539-548. [CrossRef] [PubMed]

56. Reddy, A.S.N.; Ali, G.S.; Celesnik, H.; Day, I.S. Coping with stresses: Roles of calcium- and calcium/calmodulin-regulated gene expression. Plant Cell 2011, 23, 2010-2032. [CrossRef] [PubMed]

57. Du, L.; Yang, T.; Puthanveettil, S.V.; Poovaiah, B.W. Decoding of calcium signal through calmodulin: Calmodulin-binding proteins in plants. Signal. Commun. Plants 2011, 10, 177-233.

58. Liu, Q.; Li, X.; Yan, S.; Yu, T.; Yang, J.; Dong, J.; Zhang, S.; Zhao, J.; Yang, T.; Mao, X.; Zhu, X.; Liu, B. OsWRKY 67 positively regulates blast and bacteria blight resistance by direct activation of $P R$ genes in rice. BMC Plant Biol. 2018, 18, 257. [CrossRef]

59. Peng, Y.; Bartley, L.E.; Chen, X.; Dardick, C.; Chern, M.; Ruan, R.; Canlas, P.E.; Ronald, P.C. OsWRKY62 is a negative regulator of basal and Xa21-mediated defense against Xanthomonas oryzae pv. oryzae in rice. Mol. Plant 2008, 1, 446-458. [CrossRef]

60. Chujo, T.; Miyamoto, K.; Shimogawa, T.; Shimizu, T.; Otake, Y.; Yokotani, N.; Nishizawa, Y.; Shibuya, N.; Nojiri, H.; Yamane, H.; Minami, E.; Okada, K. OsWRKY28, a PAMP-responsive transrepressor, negatively regulates innate immune responses in rice against rice blast fungus. Plant Mol. Biol. 2013, 82, $23-37$. [CrossRef]

61. Liu, Y.; Liu, Q.; Tang, Y.; Ding, W. NtPR1a regulates resistance to Ralstonia solanacearum in Nicotiana tabacum via activating the defense-related genes. Biochem. Biophys. Res. Commun. 2019, 508, 940-945. [CrossRef] [PubMed]

62. Antoniw, J.F.; Pierpoint, W.S. Purification of a tobacco leaf protein associated with resistance to virus infection. Biochem. Soc. Trans. 1978, 6, 248-250. [CrossRef] [PubMed]

63. Alexander, D.; Goodman, R.M.; Gut-Rella, M.; Glascock, C.; Weymann, K.; Friedrich, L.; Maddox, D.; Ahl-Goy, P.; Luntz, T.; Ward, E. Increased tolerance to two oomycete pathogens in transgenic tobacco expressing pathogenesis-related protein 1a. Proc. Natl. Acad. Sci. USA 1993, 90, 7327-7331. [CrossRef] [PubMed]

64. Chin, K.; DeFalco, T.A.; Moeder, W.; Yoshioka, K. The Arabidopsis cyclic nucleotide-gated ion channels AtCNGC2 and AtCNGC4 work in the same signaling pathway to regulate pathogen defense and floral transition. Plant Physiol. 2013, 163, 611-624. [CrossRef] [PubMed]

65. Zhang, W.; Dong, C.; Zhang, Y.; Zhu, J.; Dai, H.; Bai, S. An apple cyclic nucleotide-gated ion channel gene highly responsive to Botryosphaeria dothidea infection enhances the susceptibility of Nicotiana benthamiana to bacterial and fungal pathogens. Plant Sci. 2018, 269, 94-105. [CrossRef] [PubMed]

66. Yu, I.C.; Parker, J.; Bent, A.F. Gene-for-gene disease resistance without the hypersensitive response in Arabidopsis dnd1 mutant. Proc. Natl. Acad. Sci. USA 1998, 95, 7819-7824. [CrossRef]

67. Jurkowski, G.I.; Smith, R.K., Jr.; Yu, I.C.; Ham, J.H.; Sharma, S.B.; Klessig, D.F.; Fengler, K.A.; Bent, A.F. Arabidopsis DND2, a second cyclic nucleotide-gated ion channel gene for which mutation causes the defense, no death phenotype. Mol. Plant Microbe Interact. 2004, 17, 511-520. [CrossRef] [PubMed]

68. Kim, M.G.; da Cunha, L.; McFall, A.J.; Belkhadir, Y.; DebRoy, S.; Dangl, J.L.; Mackey, D. Two Pseudomonas syringae type III effectors inhibit RIN4-regulated basal defense in Arabidopsis. Cell 2005, 121, 749-759. [CrossRef] 
69. Liu, J.; Elmore, J.M.; Fuglsang, A.T.; Palmgren, M.G.; Staskwicz, B.J.; Coaker, G. RIN4 functions with plasma membrane $\mathrm{H}^{+}$-ATPases to regulate stomatal apertures during pathogen attack. PLoS Biol. 2009, 7, e1000139. [CrossRef]

70. Yamada, K.; Yamashita-Yamada, M.; Hirase, T.; Fujiwara, T.; Tsuda, K.; Hiruma, K.; Saijo, Y. Danger peptide receptor signaling in plants ensures basal immunity upon pathogen-induced depletion of BAK1. EMBO J. 2016, 35, 46-61. [CrossRef]

71. Yasuda, S.; Okada, K.; Saijo, Y. A look at plant immunity through the window of the multitasking coreceptor BAK1. Curr. Opin. Plant Biol. 2017, 38, 10-18. [CrossRef] [PubMed]

72. Ricci-Azevedo, R.; Roque-Barreira, M.C.; Gay, N.J. Targeting and recognition of Toll-like receptors by plant and pathogen pectins. Front. Immunol. 2017, 8, 1820. [CrossRef]

73. Salmeron, J.M.; Oldroyd, G.E.; Rommens, C.M.; Scofield, S.R.; Kim, H.S.; Lavelle, D.T.; Dahlbeck, D.; Staskawicz, B.J. Tomato Prf is a member of the leucine-rich repeat class of plant disease resistance genes and lies embedded within the Pto kinase gene cluster. Cell 1996, 86, 123-133. [CrossRef]

74. Gao, Y.L.; Wang, B.W.; Xu, Z.L.; Li, M.Y.; Song, Z.B.; Li, W.Z.; Li, Y.P. Tobacco serine/threonine protein kinase gene NrSTK enhances black shank resistance. Genet. Mol. Res. 2015, 14, 16415-16424. [CrossRef]

75. Yang, X.; Deng, F.; Ramonell, K.M. Receptor-like kinases and receptor-like proteins: Keys to pathogen recognition and defense signaling in plant innate immunity. Front. Biol. 2012, 7, 155-166. [CrossRef]

76. Underwood, W.; Somerville, S.C. Phosphorylation is required for the pathogen defense function of the Arabidopsis PEN3 ABC transporter. Plant Signal. Behav. 2017, 12, e1379644. [CrossRef] [PubMed]

77. Meng, D.; Dubin, M.; Zhang, P.; Osborne, E.J.; Stegle, O.; Clark, R.M.; Nordborg, M. Limited contribution of DNA methylation variation to expression regulation in Arabidopsis thaliana. PLoS Genet. 2016, 12, e1006141. [CrossRef] [PubMed]

78. Shen, Y.; Zhang, J.; Liu, Y.; Liu, S.; Liu, Z.; Duan, Z.; Wang, Z.; Zhu, B.; Guo, Y.L.; Tian, Z. DNA methylation footprints during soybean domestication and improvement. Genome Biol. 2018, 19, 128. [CrossRef] [PubMed]

79. Zilberman, D.; Gehring, M.; Tran, R.K.; Ballinger, T.; Henikoff, S. Genome-wide analysis of Arabidopsis thaliana DNA methylation uncovers an interdependence between methylation and transcription. Nat. Genet. 2007, 39, 61-69. [CrossRef] [PubMed]

80. Gent, J.I.; Ellis, N.A.; Guo, L.; Harkess, A.E.; Yao, Y.; Zhang, X.; Dawe, R.K. CHH islands: De novo DNA methylation in near-gene chromatin regulation in maize. Genome Res. 2013, 23, 628-637. [CrossRef] [PubMed]

81. Ramirez-Prado, J.S.; Abulfaraj, A.A.; Rayapuram, N.; Benhamed, M.; Hirt, H. Plant immunity: From signaling to epigenetic control of defense. Trends Plant Sci. 2018, 23, 833-844. [CrossRef] [PubMed]

82. Diaz-Pendon, J.A.; Li, F.; Li, W.X.; Ding, S.W. Suppression of antiviral silencing by cucumber mosaic virus $2 \mathrm{~b}$ protein in Arabidopsis is associated with drastically reduced accumulation of three classes of viral small interfering RNAs. Plant Cell 2007, 19, 2053-2063. [CrossRef] [PubMed]

83. Yan, F.; Zhang, H.M.; Adams, M.J.; Yang, J.; Peng, J.J.; Antoniw, J.F.; Zhou, Y.J.; Chen, J.P. Characterization of siRNAs derived from Rice stripe virus in infected rice plants by deep sequencing. Arch. Virol. 2010, 155, 935-940. [CrossRef] [PubMed]

84. Dowen, R.H.; Pelizzola, M.; Schmitz, R.J.; Lister, R.; Dowen, J.M.; Nery, J.R.; Dixon, J.E.; Ecker, J.R. Widespread dynamic DNA methylation in response to biotic stress. Proc. Natl. Acad. Sci. USA 2012, 109, 2183-2191. [CrossRef] [PubMed]

(C) 2019 by the authors. Licensee MDPI, Basel, Switzerland. This article is an open access article distributed under the terms and conditions of the Creative Commons Attribution (CC BY) license (http://creativecommons.org/licenses/by/4.0/). 